\title{
Notes on the variability of reflected inner core phases
}

\author{
Dunzhu Li · Daoyuan Sun · Don Helmberger
}

Received: 28 December 2013/Accepted: 14 April 2014/Published online: 8 August 2014

(C) The Seismological Society of China, Institute of Geophysics, China Earthquake Administration and Springer-Verlag Berlin Heidelberg 2014

\begin{abstract}
Recent events beneath Central America have produced excellent sets of inner core reflection (PKiKP phase) at high frequency recorded by USArray ranging from $18^{\circ}$ to $30^{\circ}$. However, the amplitude of this phase displays considerable scatter with a factor of six or more. Such scatter has been attributed to upper-mantle scattering and the Inner Core Boundary (ICB) in combination. Here, we show that neighboring events share upper-mantle scatterers beneath the receivers, and their ratio allows a clearer image of deep earth structure. After confirming some of the measured variation is indeed due to deep structure, we stacked nearby traces to reduce fine scale variations which are mostly due to shallow structure. Then, the remaining relatively large scale variation pattern of PKiKP phase is caused by the inner core boundary, as demonstrated by numerical experiments. After migration of data to the ICB, we observe a consistent image. We find such a pattern can be explained by a patch of mushy material of a few kilometers high where the material changes gradually from that of the outer core to that of the inner core.
\end{abstract}

\section{Introduction}

The solidification process at the Inner Core Boundary (ICB) strongly influences the Earth's magnetic field, and

D. Li $(\bowtie) \cdot$ D. Helmberger

Seismological Laboratory, California Institute of Technology,

Pasadena, CA 91125, USA

e-mail: lidunzhu@gmail.com

D. Sun

Department of Earth Sciences, University of Southern California, Los Angeles, CA 90089, USA becomes of key importance to the geodynamo. Laboratory experiments indicate that a thin mushy layer forms at the boundary between a fluid and the growing solid, and Shimizu et al. (2005) suggests such a zone should exist at the ICB depending on the properties of the light element present. Such a layer is likely to be highly variable given the dynamic circulation involved. Thus, seismologists have been addressing properties of this interface with an array of interesting observations. Most detailed seismic studies of the ICB involve the various branches of the PKP system and, in particular, the differential times between PKiKP and PKIKP (Fig. 1), by Poupinet et al. (1983), Cormier and Choy (1986). Hemispheric difference became well mapped more recently, i.e., Niu and Wen (2001), Wen and Niu (2002) and Waszek et al. (2011). Short wavelength variations on the ICB based on these phases are discussed by Cao et al. (2007) along with possible temporal variation which is also discussed in an earlier paper by Wen (2006). Because PKiKP is essentially at critical angle at ranges where PKIKP is also observed $\left(130^{\circ}-140^{\circ}\right)$, it is not sensitive to density changes across the ICB, i.e., Cormier and Richards (1977) and Song and Helmberger (1992). The ICB has also been investigated at small distances (precritical angles) where the amplitude ratio of PKiKP to PcP from the LASA array was used to estimate the density jump Engdahl et al. (1970). One of the first attempts to model the fine-structure of the ICB was by Cummins and Johnson (1988) where they demonstrated that the PKiKP precritical angle reflections will be greatly reduced by adding a transition zone. Thus, they concluded that the few existing measurements indicated a very sharp interface, less than $2 \mathrm{~km}$. A number of global studies followed, i.e., Shearer and Masters (1990), attempting to further constrain the density jump at the ICB. More recently, short-period arrays have provided more definitive measurements of this 


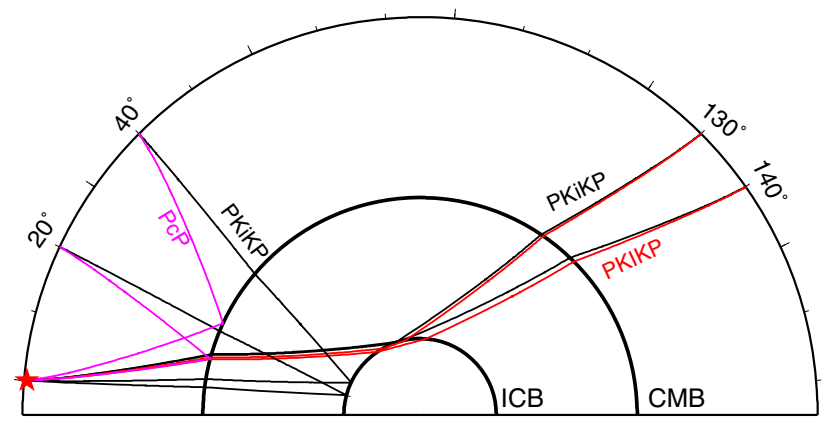

Fig. 1 Diagram of example geometrical ray paths for the two main phases used in this study: PKiKP (black) and PcP (magenta). Ray paths for PKiKP (black) and PKIKP (red) at critical angle ranges $\left(130-140^{\circ}\right)$ are also displayed

ratio, Kawakatsu (2006), and papers by Koper (2004), Koper and Dombrovskaya (2005), involving both differential times and amplitudes. They proposed a density jump of $0.525 \pm 0.239\left(\mathrm{~g} / \mathrm{cm}^{3}\right)$ where they attempted to establish a meaningful error estimate. This proves difficult but their studies and others suggest that earthquake radiation
Table 1 Events that contain visible PKiKP phases after filtering to $1-3 \mathrm{~Hz}$

\begin{tabular}{lllccc}
\hline Origin time & \multicolumn{2}{c}{ Epicentral location } & Depth $(\mathrm{km})$ & $M_{\mathrm{w}}$ \\
\cline { 2 - 4 } & Lat. $\left(^{\circ}\right)$ & Long. $\left(^{\circ}\right)$ & & \\
\hline 2006-12-03 20:52:15.73 & 14.013 & -91.342 & 61.0 & 5.98 \\
2007-06-08 13:32:02.20 & 13.769 & -90.895 & 65.0 & 5.83 \\
2007-07-06 01:09:18.48 & 16.495 & -93.784 & 121.1 & 6.06 \\
2007-07-23 22:30:08.45 & 14.381 & -90.954 & 113.0 & 5.50 \\
2008-11-15 23:03:16.59 & 13.072 & -88.927 & 48.0 & 5.81 \\
2009-01-17 02:57:31.59 & 15.742 & -92.605 & 174.1 & 5.82 \\
2009-04-27 16:46:27.49 & 16.941 & -99.568 & 33.3 & 5.83 \\
2009-05-03 16:21:45.75 & 14.618 & -91.252 & 108.0 & 6.28 \\
$2009-11-26$ & $19: 08: 11.19$ & 13.467 & -90.051 & 55.0 & 5.95 \\
\hline
\end{tabular}

pattern effects are small (with corrections less than $25 \%$ ) since the take-off angles for PcP and PKiKP are similar, see Fig. 1. Radiation pattern effects have also been addressed by Tkalcic et al. (2010), in which they argue that differential radiation amplitudes between PcP and PKiKP can probably be neglected. They also argue that much

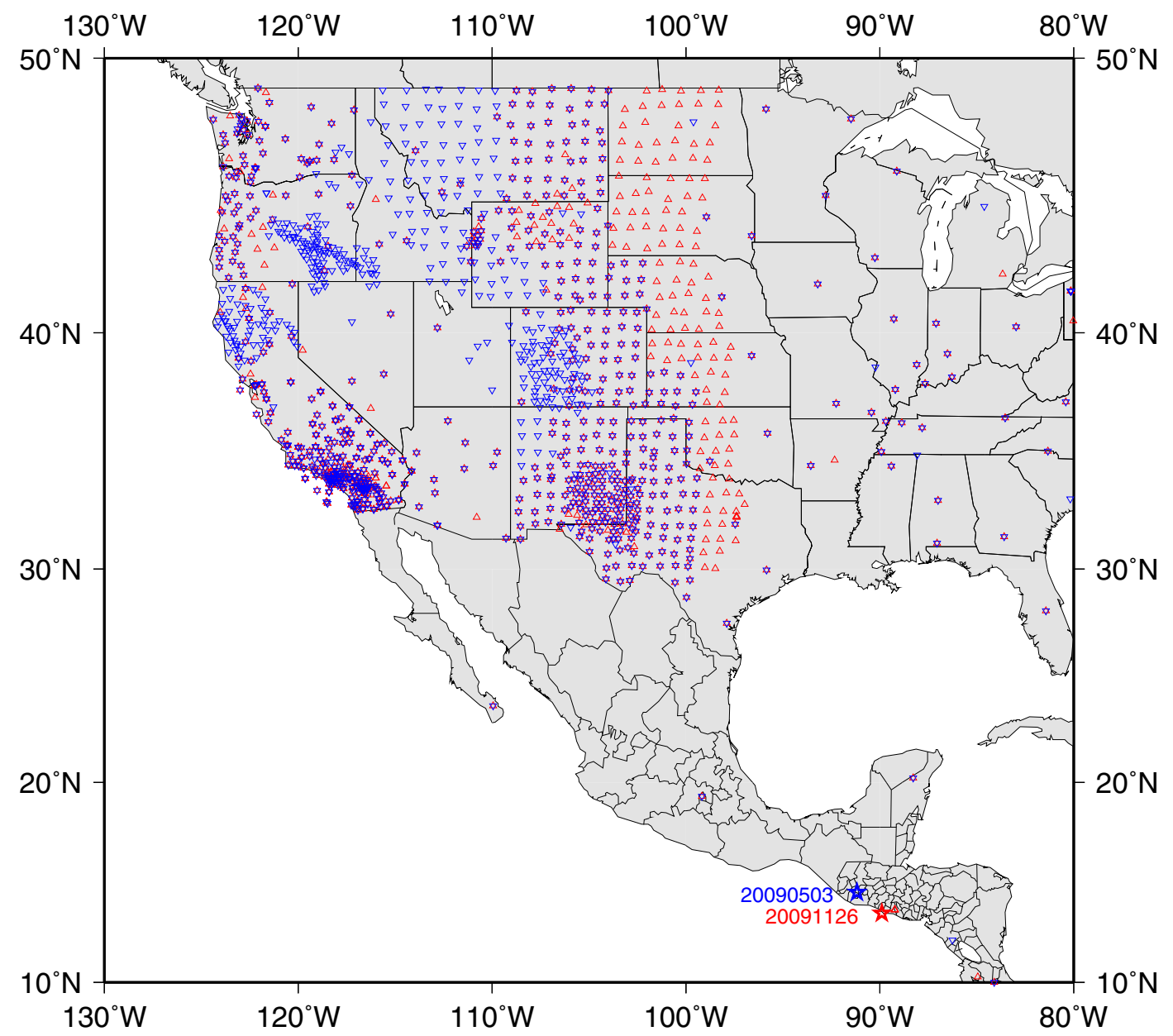

Fig. 2 Locations of two events (20090503 and 20091126, denoted as stars) and their corresponding USArray recording stations indicated by blue and red color, respectively 
a

20091126 Velocity BHZ

Time(s)

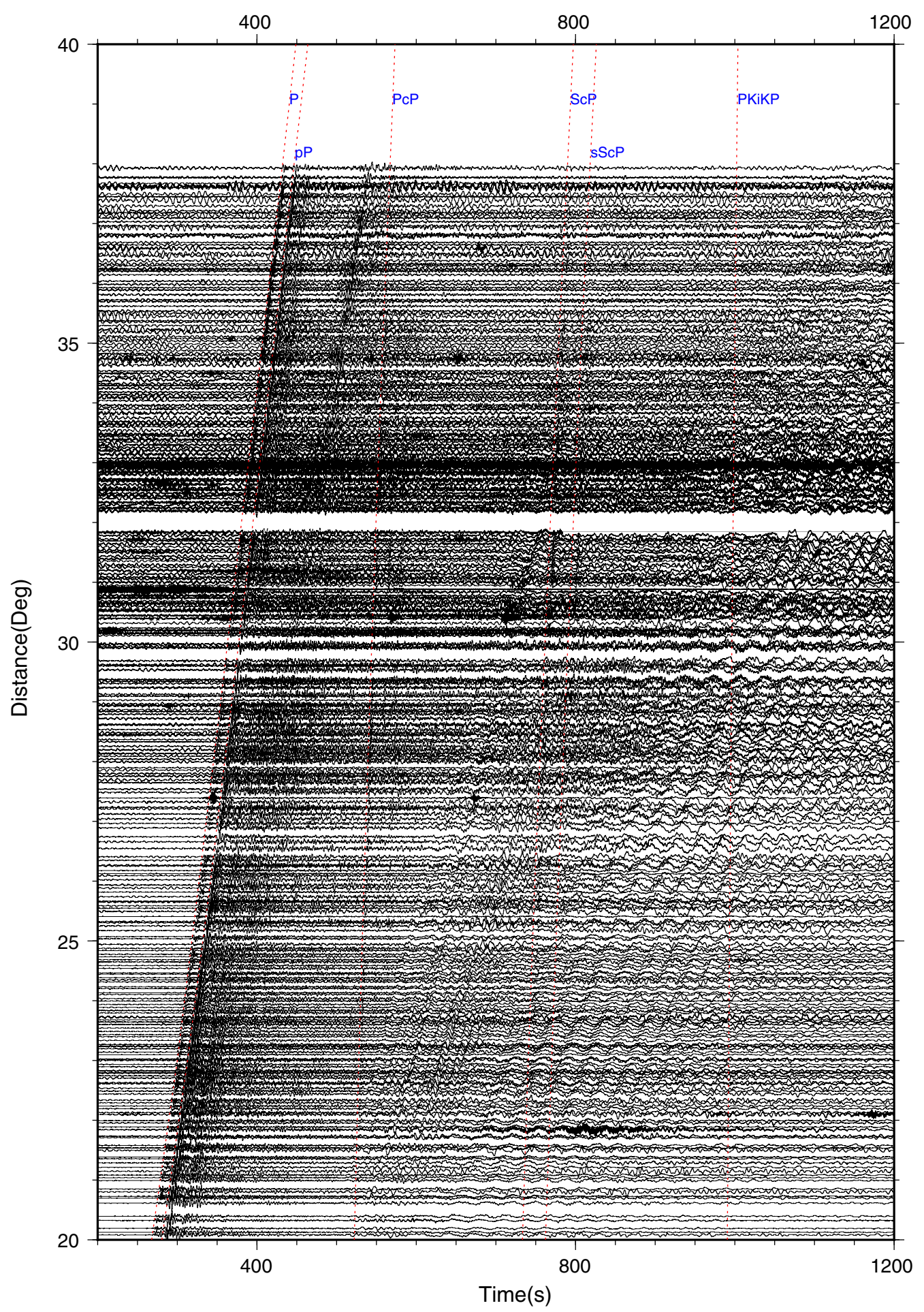

Fig. 3 a Broadband velocity seismogram for 20091126 event plotted as a record section. b Same section but applying bandpass filter from 1 to $3 \mathrm{~Hz}$ to enhance the PKiKP phase 


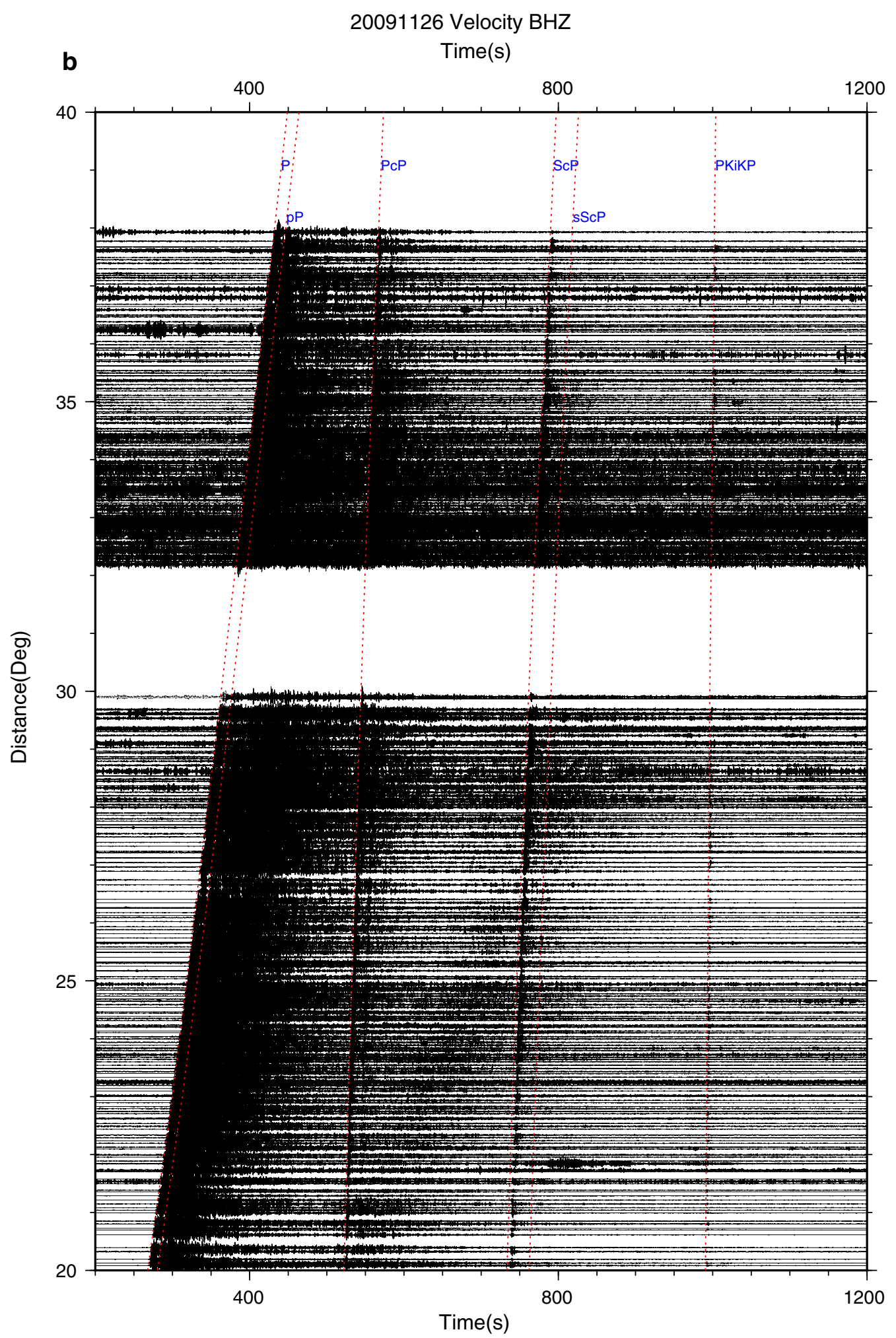

Fig. 3 continued

larger variations of this ratio are produced by path effects approaching the individual stations, and question the usefulness of these ratio measurements. They found that a deep event beneath the Kuril Islands, as recorded on the J-Array (Japan), had a negative correlation between the identification of PcP and PKiKP, i.e., if one can detect 


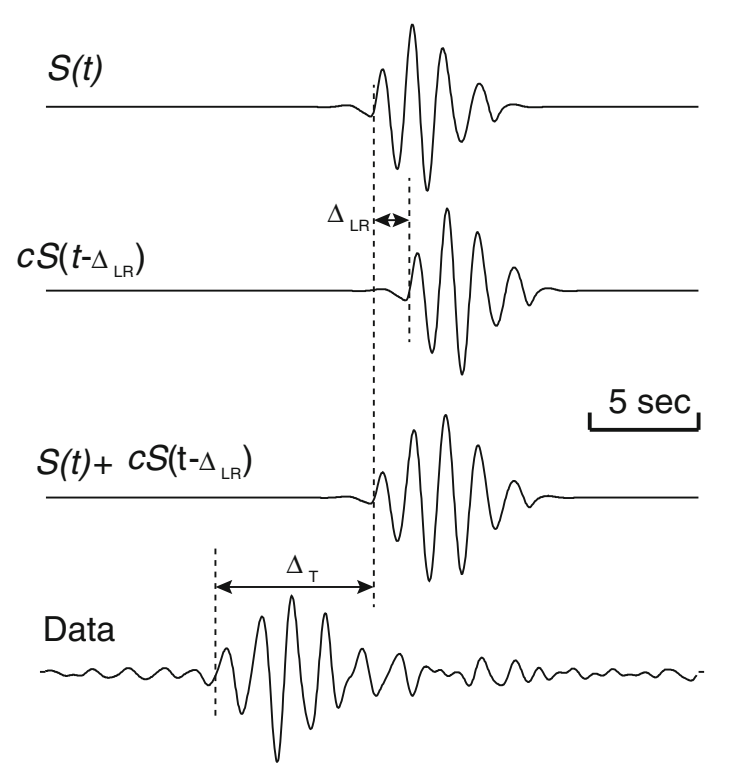

Fig. 4 Illustration of the multi-path detector (MPD) method on highfrequency data. The top trace $S(t)$ is the prediction from reference model, i.e., PREM. The MPD depends on the choice of the source time function (STF). We first examined the data and picked the record with the simplest waveform, which was then used to cross-correlate and align other data at distance range $18^{\circ}-23^{\circ}$. Then we stacked those aligned data which have high cross-correlation coefficient to form the STF. The bottom trace shows the data, which is more complicate than the $S(t)$. To simulate the data, we add the scattering contributions (same waveform as $S(t)$, but with an adjustable time shift $\Delta_{\mathrm{LR}}$ and adjustable amplitude factor $c$ ) to the reference synthetic. The best cross correlation between the data and simulated waveform is searched. The time difference between the simulated waveform to the data is defined as $\Delta_{\mathrm{T}}$

clean PcP, the PKiKP phase is in the noise and vice versa. They explain this feature by adding an upper-mantle heterogeneous layer with vertically elongated perturbations (exponential auto correlation random media, with vertical scales $100 \mathrm{~km}$ and horizontal scale $2 \mathrm{~km}, 5 \%$ RMS in $\mathrm{P}$-velocity). Thus, the difference in the upcoming ray paths allows one phase to be enhanced but not the other. They suggest that the presence of the slab along the path to Japan could be the cause of such a layer. Observations from the oceanic direction appear not to be affected as much Kawakatsu (2006). Still more recently, Dai et al. (2012) use the rapid variation in the $\mathrm{PKiKP} / \mathrm{PcP}$ ratio to map out a box-car structure, $14 \mathrm{~km}$ high with a horizontal length of about 3 degrees, as observed on the dense Hi-Net Array covering Japan.

In short, there appears to be considerable evidence for fluctuations in the pre critical angle $\mathrm{PKiKP} / \mathrm{PcP}$ ratio as discussed above. This is probably highly situation-specific as suggested by Adushkin and Ovchinnikov (2004). Here, we will investigate these phases as observed on the USArray and apply our newly developed simulation code, Li et al. (2014), to address many causes for complexities at these high frequencies. Because both the data and simulations are relatively complicated, we have added two appendixes. The first involves automated processing of waveforms that display a mixture of noise and scattering effects from deep heterogeneities. The second treats the simulation of short-period waveforms and the placement of scattering boxes at the source region and receiver region. The latter serves as a primer to previous simulation efforts.

\section{Data and processing}

Detecting the PKiKP phase at precritical angles is difficult because of its small amplitude. However, we have found a few events beneath Central America in the search window 2005-2012 displaying this phase, see Table 1. We studied the two events with the largest number of visual detections which happened to be within $1.6^{\circ}(<180 \mathrm{~km})$ of each other, see Fig. 2. Note that because the TA array is marching eastward, the two events have limited number of common stations, which, however, prove to be particularly important. The broadband record section of the 20091126 event is displayed in Fig. 3a where PKiKP cannot be identified. Observations from the 20090503 event display similar characteristics as displayed in Fig. 20. The main difficulty in observing this phase is the noise produced by regional propagating crustal waves that bury PKiKP in broadband record sections, although other core phases $\mathrm{PcP}, \mathrm{ScP}$, and $\mathrm{PcS}$ are still visible along with their depth phases. Filtering between 1 and $3 \mathrm{~Hz}$ produces the clearest view of PKiKP (Fig. 3b) as in other studies. Unfortunately, the Earth appears amazingly complicated at these frequencies and the observed PKiKP amplitude varies spatially. To obtain properties of ICB, one must evaluate and eliminate signal contamination when the signal propagates along the paths crossing both the upper-mantle and core-mantle-boundaries.

Because our analysis involves noisy data, it becomes difficult to process. We want a method that is both flexible and can be used in stacking where alignment becomes a major issue. It appears that the Multi-Path Detector (MPD) which was initially proposed for longer period studies, Sun and Helmberger (2011), is effective in this case. This method simplifies the banana-split approximation (Helmberger and Ni 2005), and assumes that the observed complex waveform is a superposition of two simple waveforms due to multi-pathing. The two simple waveforms have the same shape, but their amplitude ratio and relative time shift $\Delta_{\mathrm{LR}}$ are adjustable parameters and are searched to maximize the fits to the data, see Fig. 4. Maps of these parameters $\left(\Delta_{\mathrm{T}}, \Delta_{\mathrm{LR}}\right.$, and amplitudes are presented in Appendix 1) with respect to PREM. With these parameters, we can stack the nearby seismograms, and investigate both the amplitude and $\Delta_{\mathrm{T}}$ in stacking results. Bootstrapping 

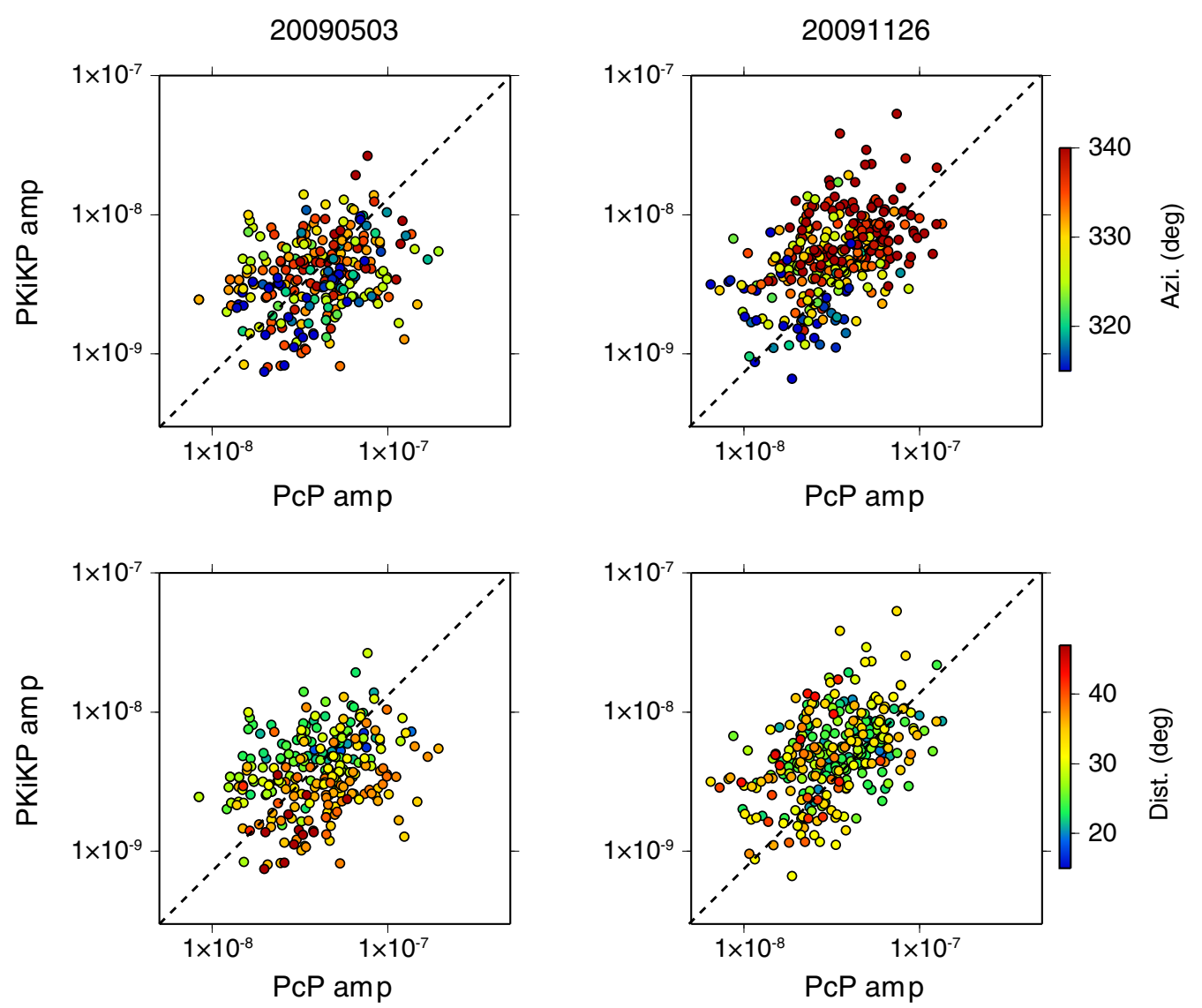

Fig. 5 PKiKP amplitude versus PcP amplitude. The symbols are color-coded by azimuth in the top row and distance in the bottom row. Note the strong scatters in the amplitudes for individual station records. Also note that the event 20090503 behaves more like PREM with the PKiKP/PcP ratio having a smaller values at large distance

procedures can be used to assess the stack reliability as applied by Sun et al. (2013).

Amplitudes between PKiKP and PcP display shotgun patterns with nearly an order-of-magnitude scatter which changes between the two events, Fig. 5. Note that the distance plot for event 20090503 shows the predicted behavior for 1D model where the ratio grows with distance as predicted in other studies, Cummins and Johnson (1988); see Appendix 2 for predicted amplitudes from PREM. The level of scatter in Fig. 5 is similar to other studies which clearly display the difficulty of using the (PKiKP/PcP) ratio as discussed in Tkalcic et al. (2010). In the next section, we will discuss some advantages of using a pair of events to help separate shallow effects from deep effects.

\section{Evidence for deep earth effects}

We begin this section with a numerical simulation (Fig. 6) assuming an upper-mantle scattering model proposed by
Tkalcic et al. (2010) but with a point source, see Appendix 2 for details. This model is not unique in that we do not know where these scatterers are located but such a model does generate strong variation in the amplitude ratio. Note that the amplitudes between the two phases are out-ofphase and greatly enhance the ratio variation. Thus, large ratio variation is easily achieved with this level of scatterers, as in Fig. 5, making the error-bars on estimating ICB density jump from ratios of individual stations huge. However, the differential behavior between two events recorded at the same station is still useful for correcting receiver effects. Note the PcP ray paths for the two events in Fig. 6 are very near to each other, so were for PKiKP ray paths. Here, the scatterers are probably stronger than for the real earth, but even so the ratio of individually phases between two events are not affected much, especially for PKiKP phase.

In short, analyzing these two nearby events is especially useful, as discussed above, to evaluate how much variation is due to deep Earth structure and how much is due to receiver structure. Thus, we select stations which recorded 
Two event seperate by 1.5 degree

a
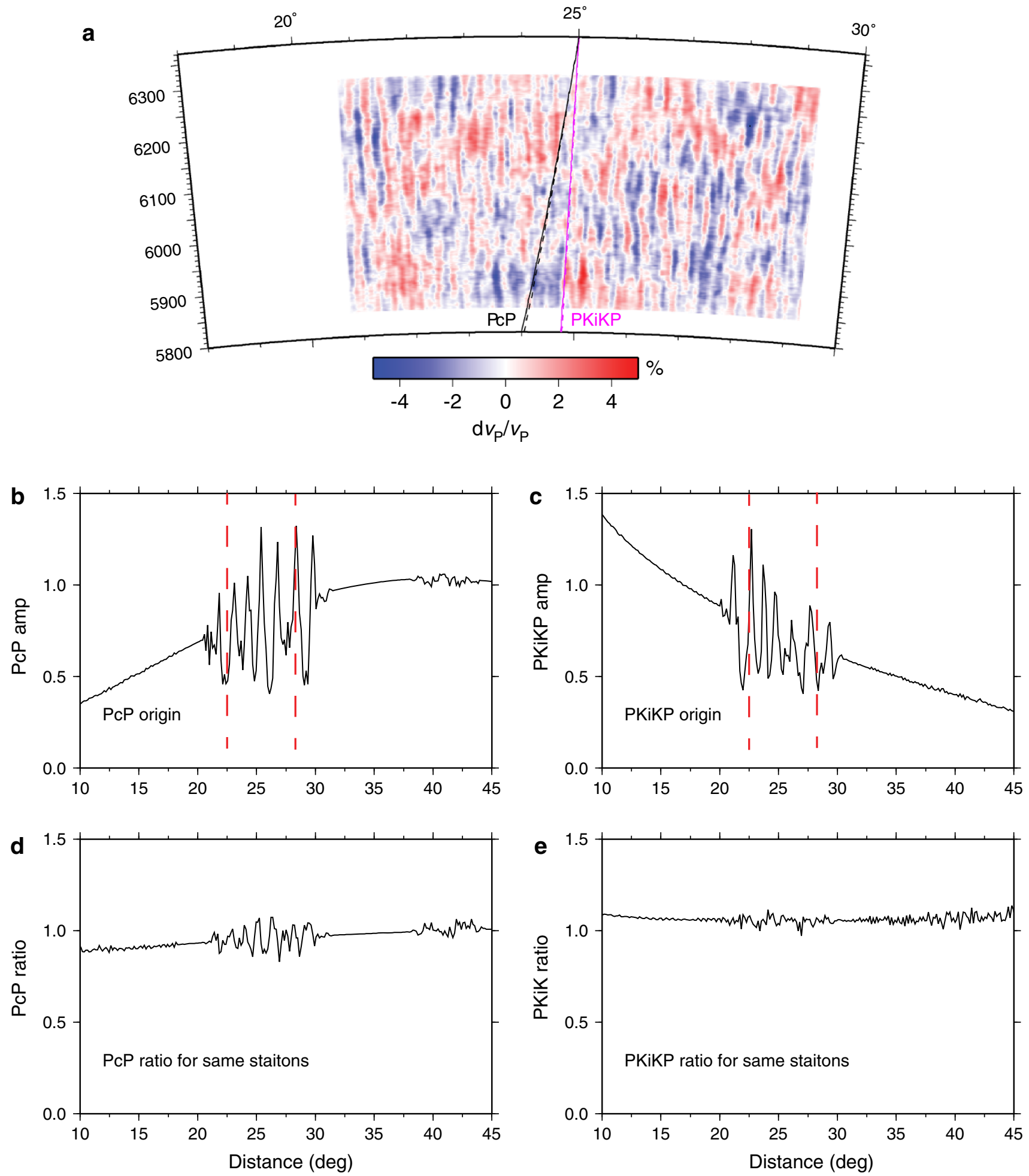

Fig. 6 Simulation of effects caused by upper-mantle heterogeneities on receiver side at the same stations for two nearby events. a Strong upper-mantle heterogeneities are included in the model. Although the two events are separated by $1.6^{\circ}$, both PcP (black) and PKiKP (magenta) ray paths (solid and dash lines) are very close in the upper mantle. The upper-mantle heterogeneities introduce strong amplitude variations on both $\mathbf{b} \mathrm{PcP}$ and $\mathbf{c} \mathrm{PKiKP}$. The d PcP ratios between two events for the same stations are relatively stable, so were for e PKiKP ratios. These ratios can be used an efficient way to remove possible strong upper-mantle effects on the high-frequency data and isolate deep earth contribution 


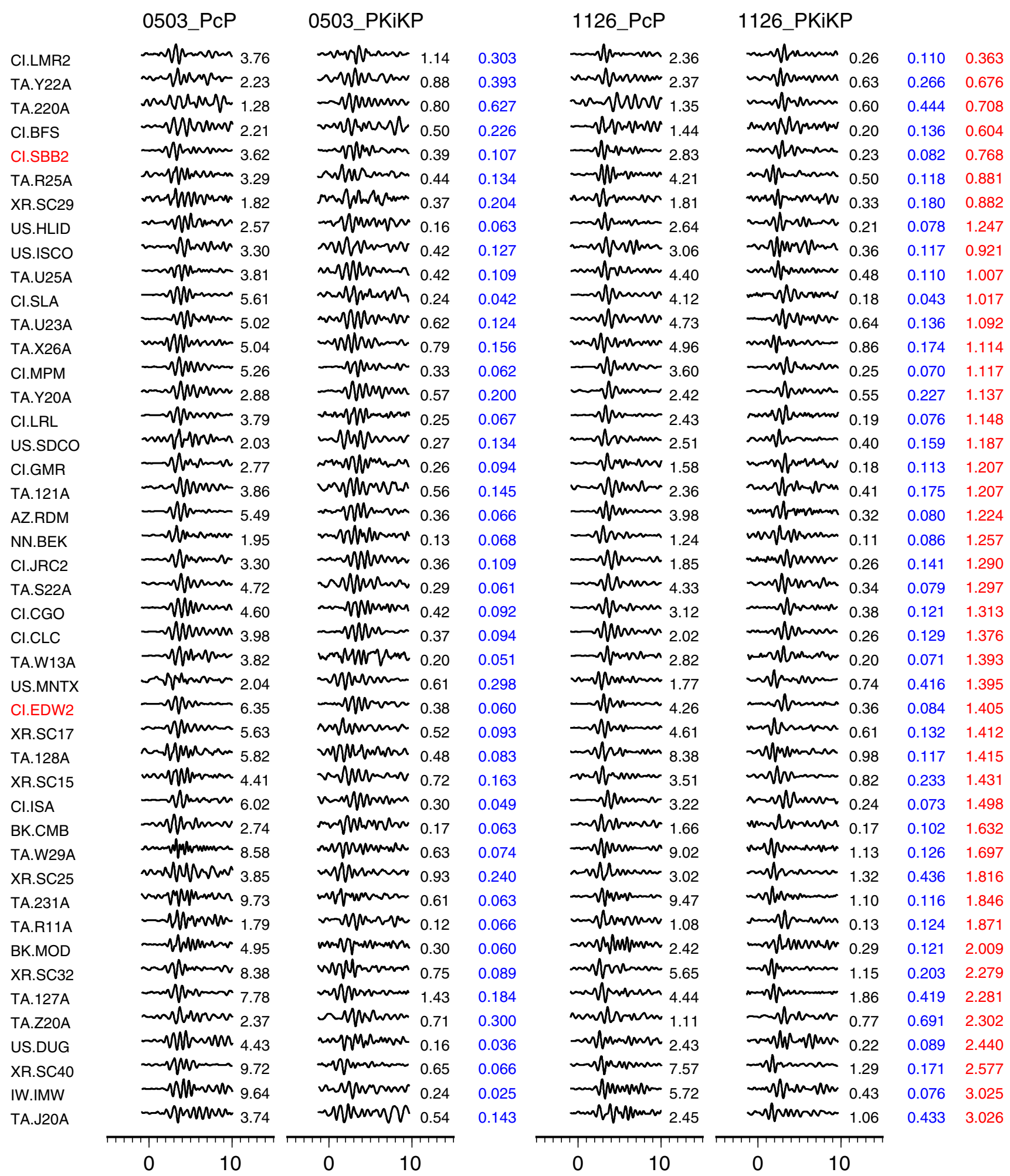

Fig. 7 Selected displacement seismograms aligned on PREM arrivals, with SNR $>2$ at $1-3 \mathrm{~Hz}$, for both PcP and PKiKP phases and recorded by both events. The black number shows the maximum absolute amplitude for each trace $(\times 1 \mathrm{E}-8 \mathrm{~m})$, the blue number shows the ratio between PKiKP and PcP for each event, and the red number indicates the ratio of the two blue number. Seismograms are ordered by the red number 

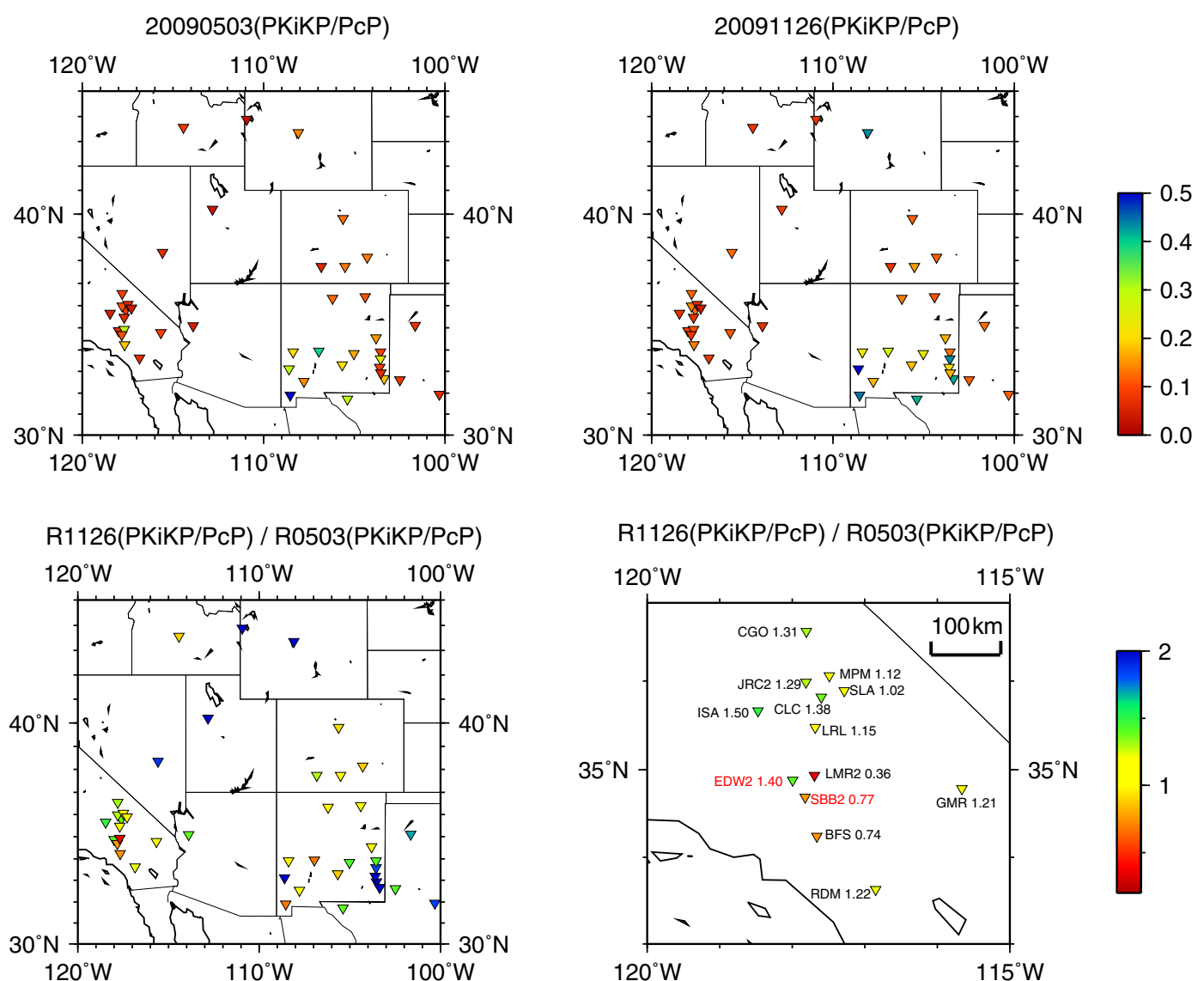

Fig. 8 In map view, top two panels show the PKiKP/PcP ratio for the two events, and bottom left panel shows ratio of ratio between the two events, with a zoom-in of CI array in bottom right panel. Note the jump of ratio of ratio at relatively close CI stations
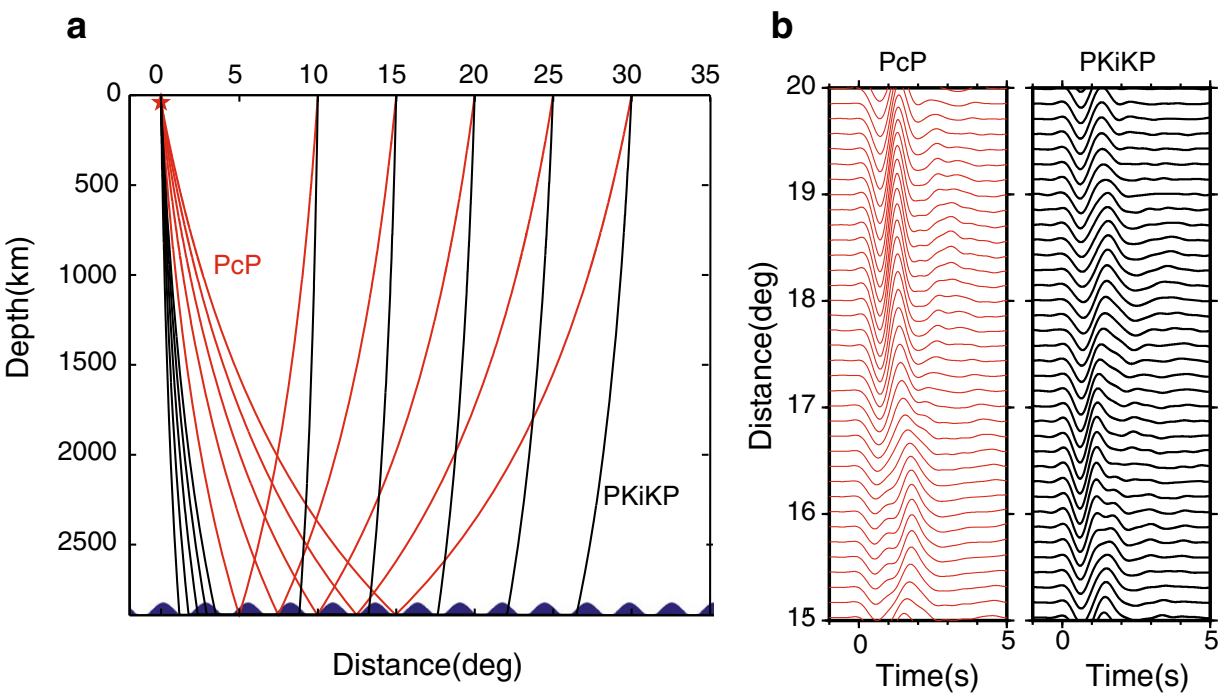

C

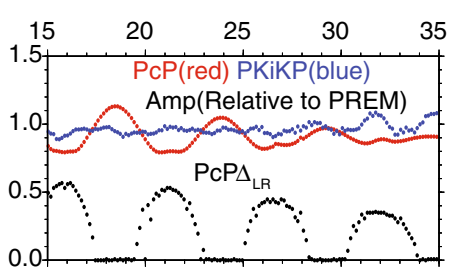

Fig. 9 Simulation of effects of the CMB structure. a The model includes sinusoids of low velocity structure at the CMB with the width of $100 \mathrm{~km}$ and the height of $100 \mathrm{~km}$. Inside the low velocity structure, the $V_{\mathrm{P}}$ anomaly is $-5 \%$. Note the PcP (red) and PKiKP (black) sample very different parts of the low velocity structure. $\mathbf{b}$ The synthetics of PcP and PKiKP for the model in (a). The low velocity structures at the CMB have much less effect on PKiKP waveforms than PcP waveforms. The PKiKP amplitudes (blue line) in (c) are almost constant. In contrast, the amplitudes of PcP (red line) display greater variations, where large $\Delta_{\mathrm{LR}}$ (black line) values correlate with smaller amplitudes. Thus, the PKiKP/ $\mathrm{PcP}$ ratio is mainly controlled by the variations in the amplitude of $\mathrm{PcP}$ in this model 

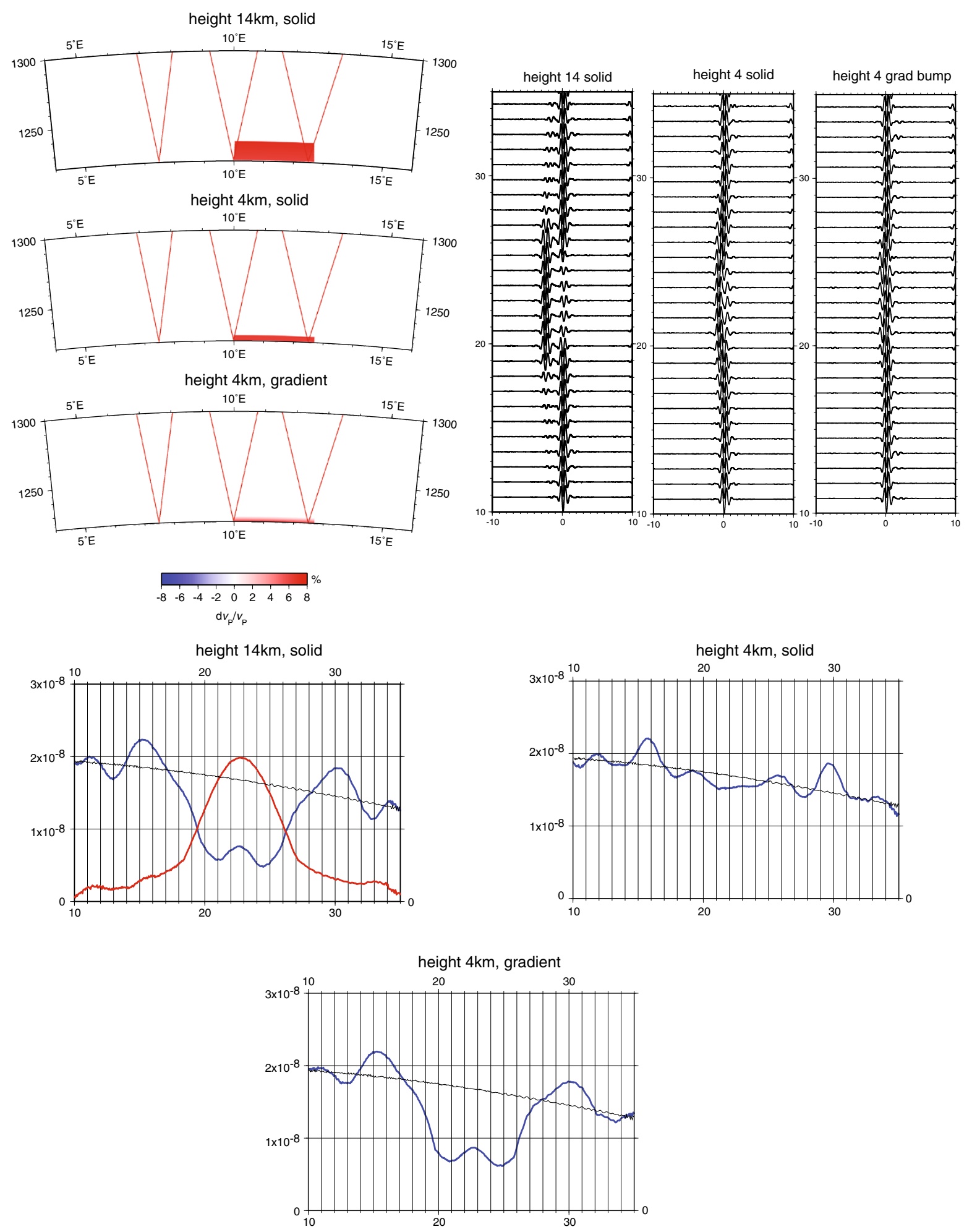

Fig. 10 Three models at ICB, where the top one contains anomalies $2.8^{\circ}$ width and $14 \mathrm{~km}$ high with inner core materials property, the second one is the same as the first one except a $4 \mathrm{~km}$ height is used, and the third one is similar to the second one except now the anomaly is a gradient transition between outer core material and inner core material. The seismograms of PKiKP for the three models are shown on the left, and the amplitude plots are shown below. Note the first model PKiKP has two arrivals, one corresponding to reflection from the top of the anomaly (precursor), shown in red, and the other is the non-optical ray reflections from ICB outside the anomaly, shown in blue. The amplitude for 1D reference model is shown in black 

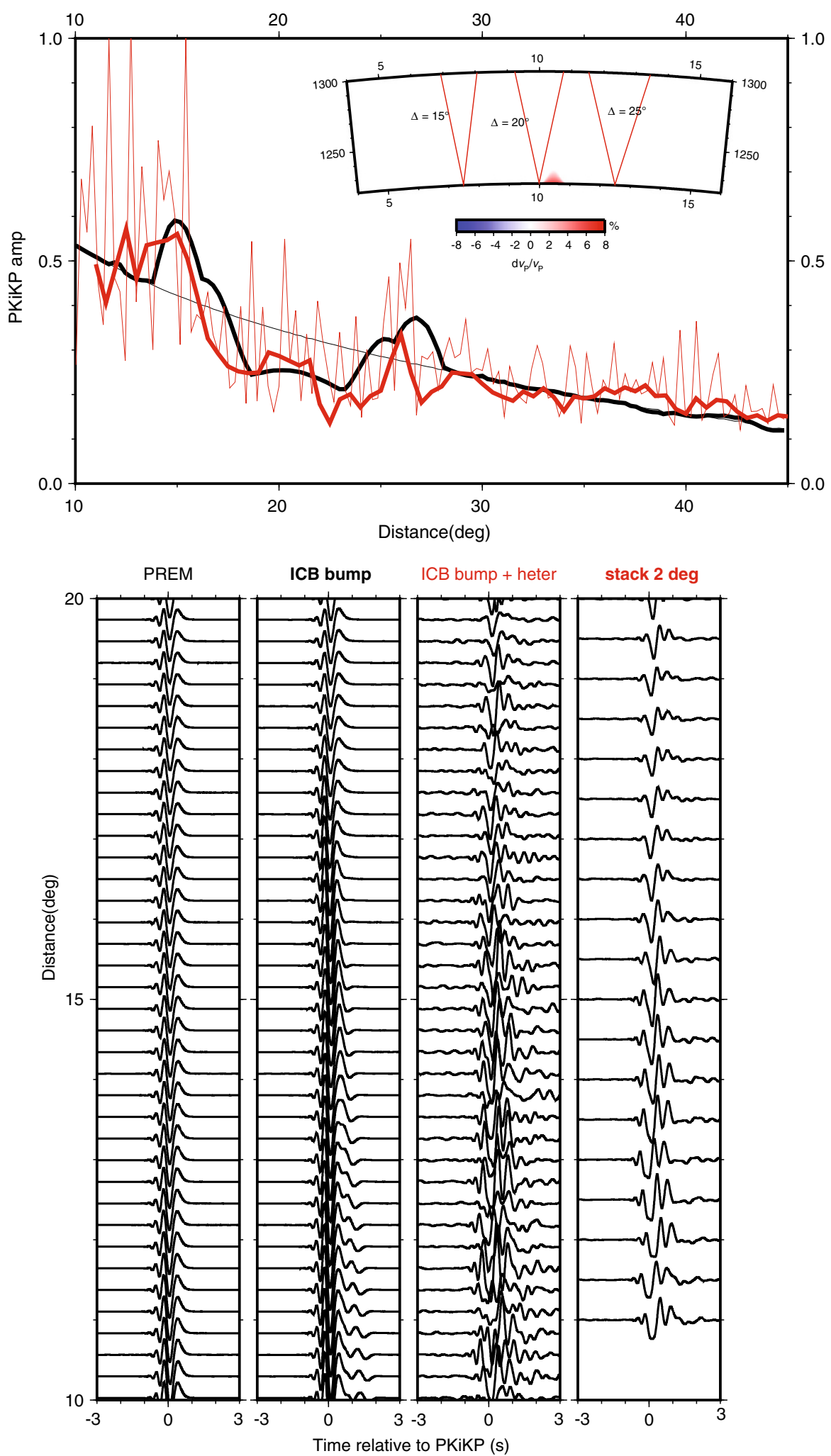

Fig. 11 Testing the stacking procedure on simulated waveforms, where influence of upper-mantle heterogeneity is greatly reduced. Three models are considered. The first one is 1D PREM, the second one added a ICB bump to the first one, and the third model added upper-mantle heterogeneity to the second model. The recorded PKiKP phase is shown on the left three record sections, and stacking of the third record section is shown in the rightmost. The amplitude of the four record section is displaced in thin line (PREM), thick dashed line (ICB bump), thin dashed line (ICB bump + heterogeneity), and red dashed line (stack $2^{\circ}$ ) respectively 
a 20091126
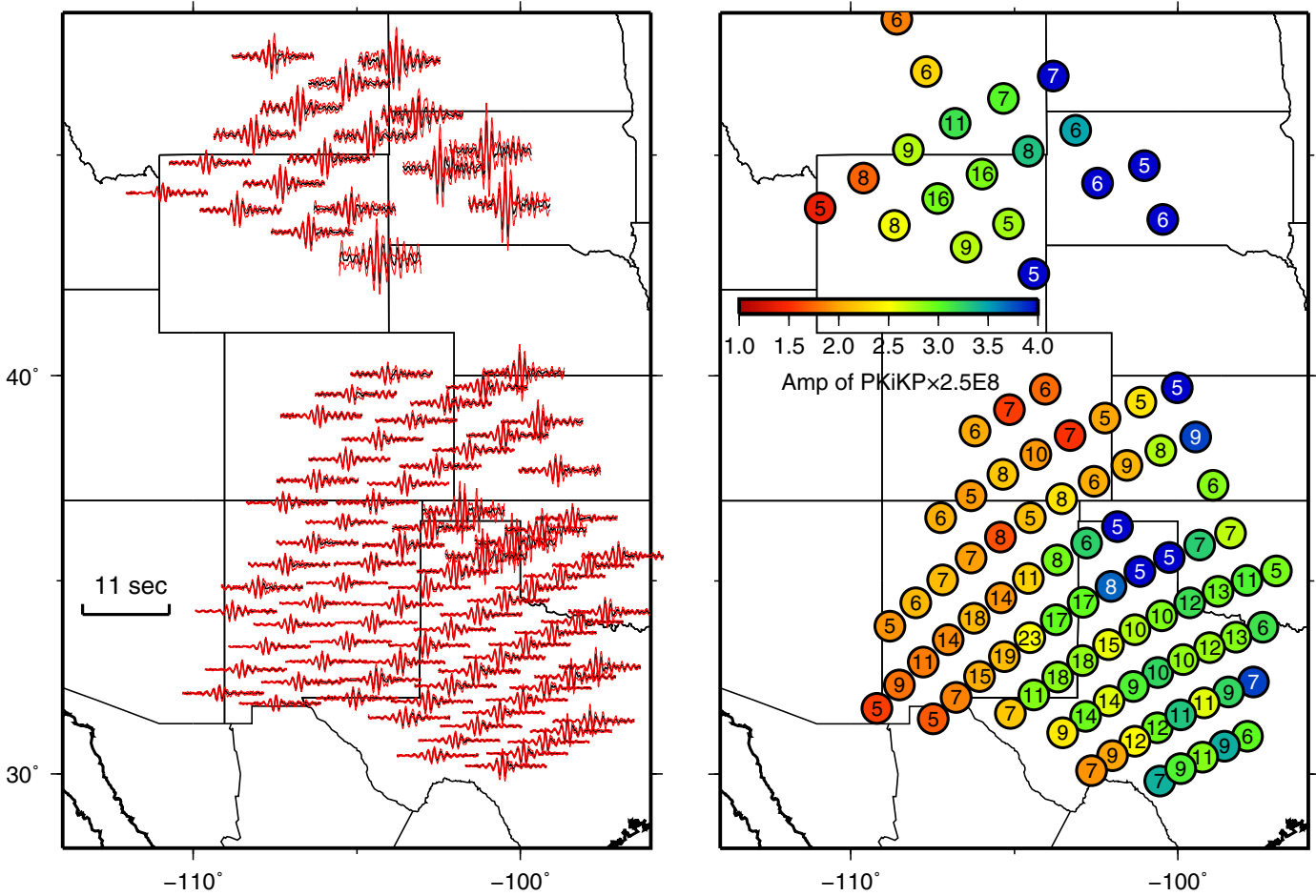

b 20090503

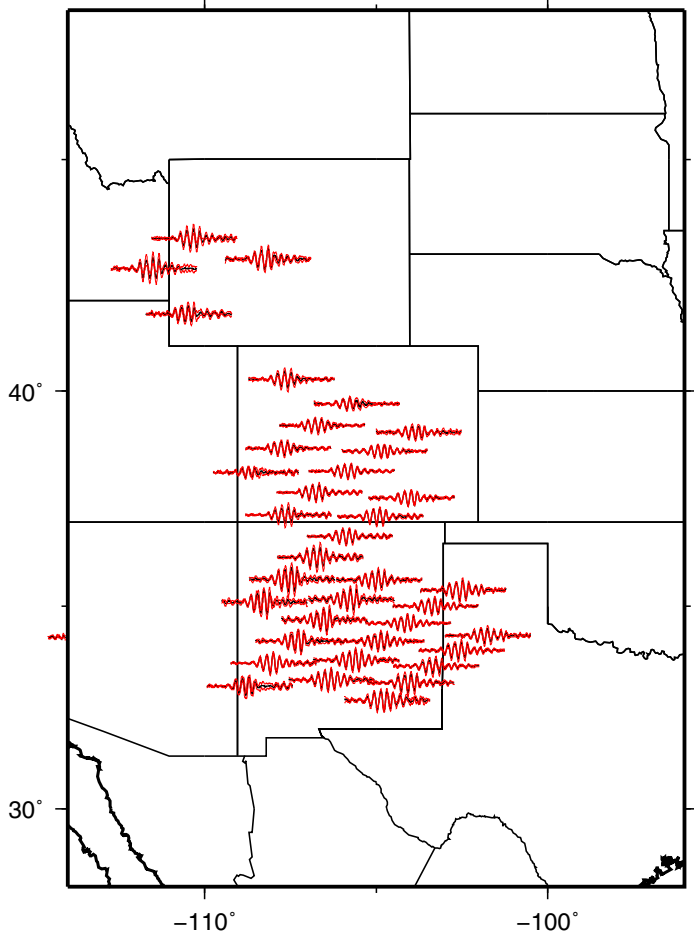

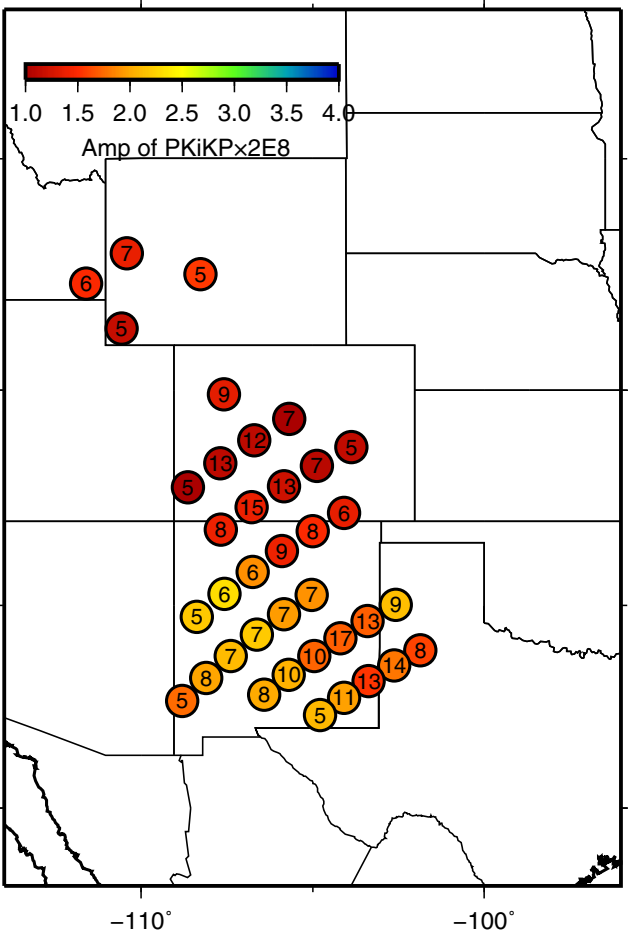

Fig. 12 Stacked PKiKP records and their amplitudes for events a 20091126 and b 20090503. Every waveform in the left column is the stacked record for the data within a circle having the radius of $120 \mathrm{~km}$. A bootstrap-resampling algorithm was applied to generate the stacked waveforms (black traces), and the waveforms with $95 \%$ confidence level are displayed in red. In the right column, the amplitudes of the stacked PKiKP waveforms are displayed in colors. The numbers inside the circle indicate the number of traces used in the stacking process 

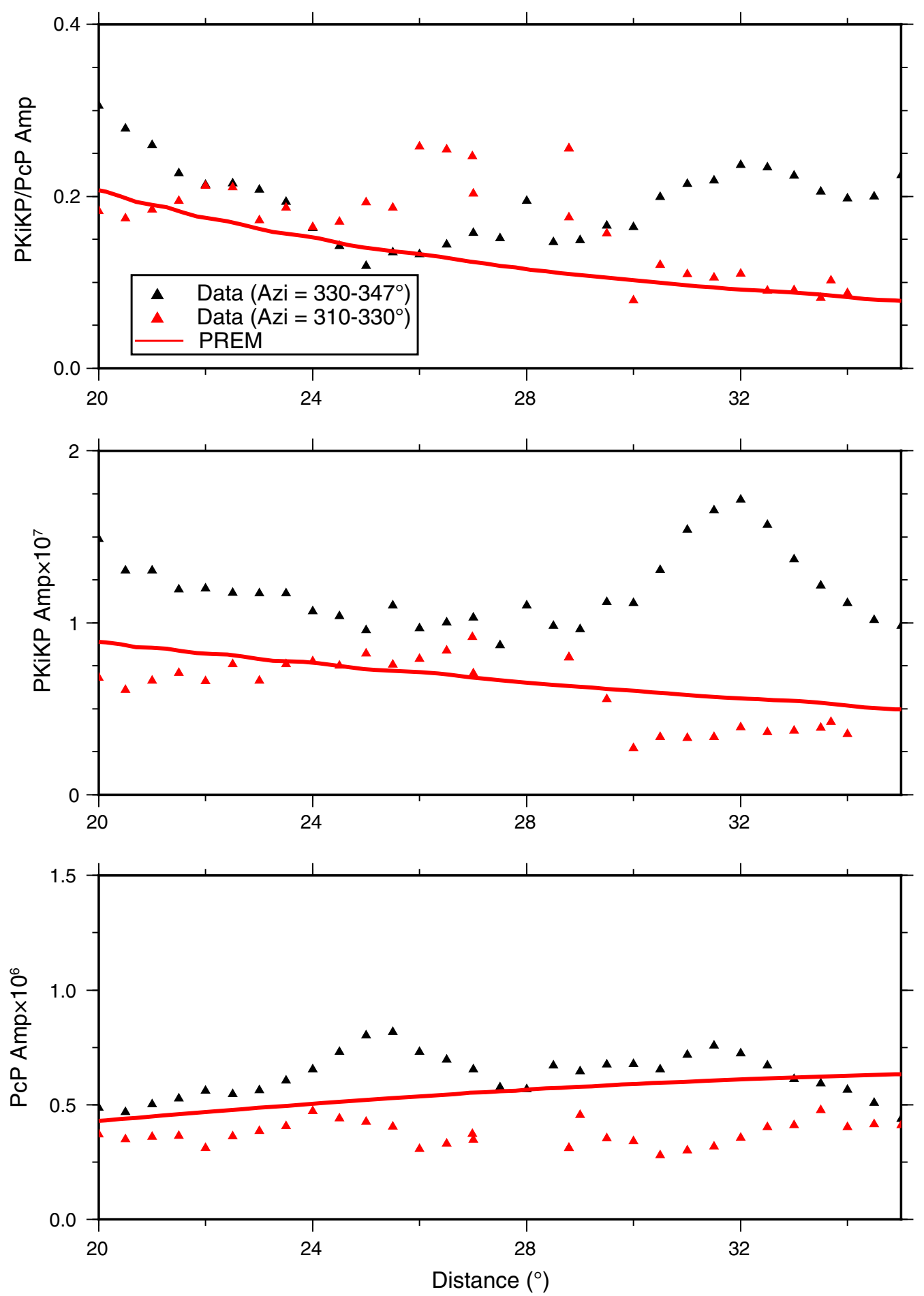

Fig. 13 The amplitudes of the stacked records along the two azimuth bins for event 20091126. From the top to the bottom: PKiKP/PcP amplitude ratio, PKiKP amplitude, and PcP amplitude. The predictions from the PREM models are displayed as red lines

clean (signal-to-noise ratio larger than two) PcP and PKiKP phases for both events, as displayed in Fig. 7 aligned on the predicted PREM travel times. Generally, for each event, the ratio $(\mathrm{PKiKP} / \mathrm{PcP}$, denoted as $\mathrm{R})$ is quite variable as noted earlier. However, on average, this ratio for first event seems to be similar to the ratio for the second event. This implies that at these stations, amplitude variations are mostly controlled by the receiver portion of the path as 


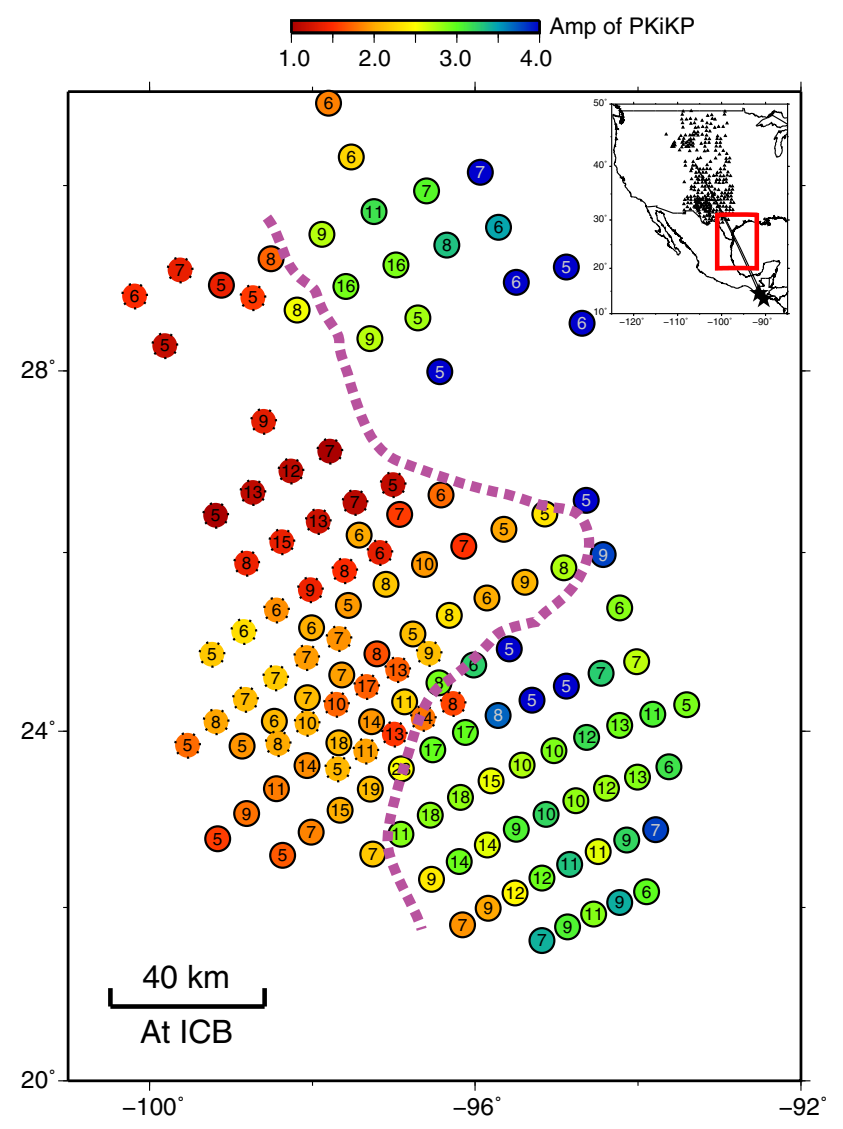

Fig. 14 The PKiKP amplitude (normalized with respect to one station) patterns in Fig. 12 migrated to the ICB. The inset displays the map of the two events and stations. The solid circles are for event 20091126, and the dashed ones are for event 20090503. The numbers inside the circle indicate the number of traces used in the stacking as in Fig. 12. The thick magenta dash line separates the region with high amplitude (green/blue color) and low amplitude (red color), which indicates possible sharp transition occurring along this line at the ICB

demonstrated by Tkalcic et al. (2010). The observation also suggests that the individual $\mathrm{PKiKP} / \mathrm{PcP}$ ratio may not be good for studying ICB because of upper-mantle scatterers. Also note that ratio of $\mathrm{PcP}$ amplitude between the two events effectively eliminates upper-mantle influence, but still shows scatter indicating CMB is not simple.

The receiver side effects cannot be the only reason for the amplitude variation. The ratios for the two events are displayed in maps view in Fig. 8. Note that there are many neighboring stations with rather large differences. For example, if we examine more closely the ratio of the dataset in the lower left panel of Fig. 8, we see that there are distinct patterns, i.e., the apparent boundary along the New MexicoTexas border. A particularly good example of rapid change is the comparison of some of stations in the southern California (CI array). Note that the ratio displays a north-south linear pattern (lower right panel of Fig. 8). A close comparison of two stations (EDW2 and SBB2) along this boundary indicates a jump by a factor of two within a horizontal distance of less than $50 \mathrm{~km}$, see Fig. 22 for details. Such a change is difficult to explain without very sharp features in the deep earth, as demonstrated by the numerical experiment in Fig. 6. Thus, some variations of PKiKP amplitude do provide some information about deep structure.

In summary, there is evidence for amplitude variations at all boundaries including the CMB and ICB. In the next section, we present some modeling results that suggest the ICB boundary can still be investigated using the above dataset.

\section{Modeling deep structure}

In the previous section, we discussed how the upper-mantle path effects can seriously interfere with the $\mathrm{PKiKP} / \mathrm{PcP}$ ratios, especially approaching the receivers. One way to reduce the upper-mantle effects is by stacking nearby traces. We will discuss this later, but first we study the effects of CMB and ICM structure on the PKiKP phase.

We assume a wash-broad pattern anomaly at the CMB, Fig. 9. The 1D ray paths (after earth flattening) for the two phases are displayed, along with synthetic waveforms from $15^{\circ}$ to $20^{\circ}$. Note that the structure focuses PcP energy from $17^{\circ}$ to $20^{\circ}$ (concave upward) and defocuses (upward bump) near $15^{\circ}$ as displayed in Fig. 9b. In Fig. 9c, the MPD result shows the good correlation of PcP amplitude with multipathing time $\left(\Delta_{\mathrm{LR}}\right)$, where the larger the splitting the smaller the amplitude. In contrast, the PKiKP phase is less affected and the $\mathrm{PKiKP} / \mathrm{PcP}$ ratio is controlled by $\mathrm{PcP}$ as shown in the lower panel of Fig. 9c. This test demonstrates that it is relatively easy to change both the timing and amplitude of PcP without significantly changing PKiKP by having structures at the $\mathrm{CMB}$.

But the simplest way to change PKiKP may be by adding topography to the ICB as pointed out by Dai et al. (2012). For example, they examined the effects of inserting a box-like structure roughly $14 \mathrm{~km}$ high, 3 degree wide, with properties of the inner core (Fig. 10, first model). This feature produces a multi-pathed PKiKP, one from the top of box (precursor) and one from the original ICB coming from each side. Their relative strength depends on geometry with the strongest precursor occurring for the ray sampling near the center of the box. The precursor becomes small (in the noise) for box 

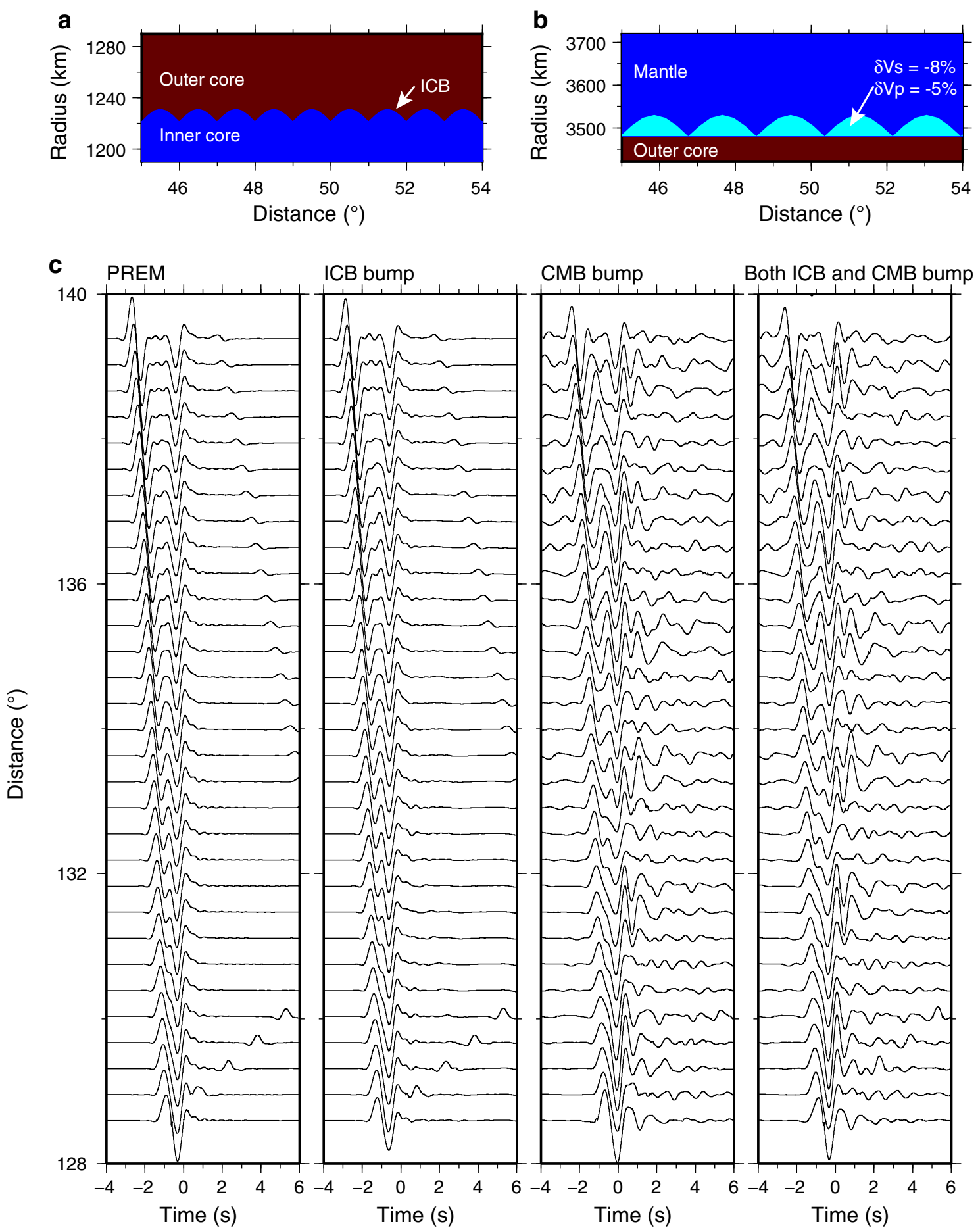

Fig. 15 Scenario study for typical structures at CMB and ICB at PKiKP critical angle ranges. $\mathbf{a}$ and $\mathbf{b}$ display the implemented structures at core boundaries. From left to right, $\mathbf{c}$ displays synthetics for PREM model, model only with ICB structure in (a), model only with CMB structure in (b), and model with both ICB and CMB structures. Note the PKiKP is not particularly sensitive to the ICB structure as the CMB structure at this distance range

widths less than about $50 \mathrm{~km}$, but still can affect the amplitude of PKiKP as displayed in Fig. 23. Reducing the height of the box to $4 \mathrm{~km}$ (Fig. 10, second model) brings the two pulses together. Note in this case, the amplitude of PKiKP is similar to 1D. However, if we let the properties of the box change gradually from outer core material on top to inner core material on bottom (Fig. 10, third model), the PKiKP amplitude can easily change by factors of two. 


\section{Results}

Stacking nearby traces reduces small scale scatter, which is mostly due to upper mantle. Figure 11 demonstrates such a scenario. We assume a target ICB anomaly (a $10 \mathrm{~km}$ high one degree wide Gaussian shape anomaly) with material gradually changing from that of outer core to that of inner core, which appears to be useful in explaining our data as shown later. Synthetics for three models and one stacked record section from $10^{\circ}$ to $20^{\circ}$ are displayed below. PREM synthetics are on the leftmost. The column next displays the effect of adding the ICB bump, which changes the PKiKP amplitude. Then adding scattering in the upper mantle produces a great deal of more variation. Finally in the rightmost record section, we stacked the third record section over a $2^{\circ}$ window, which greatly reduces the uppermantle scattering effects. More stacking produces the same decay as PREM but less revealing in recognizing features with rapid amplitude drops near $20^{\circ}$.

Based on these numerical experiments, we returned to the USArray dataset and stacked over a moving window of $1.5^{\circ}$ which generally included over eight stations. The results are displayed in Fig. 12. Here, the data are aligned on the $\Delta_{\mathrm{T}}$ shifts of each trace relative to the reference arrival time. Bootstrapping analysis as discussed by Sun et al. (2013) indicates that the stacking is stable. Generally, stacking about five stations is needed to produce a stable measurement in case of neighboring stacks that are quite similar. The station coverage for the two PASSCAL arrays contains many more traces as indicated in Fig. 12. The variation in amplitude is given in color where the strong difference in gradient occurs between the two events near the New Mexico-Texas border. Note that if this pattern was controlled by upper-mantle attenuation, both events would behave the same which is not the case. Moreover, the amplitudes of PcP do not show such feature which it should, if the PKiKP pattern was caused by attenuation. The stacked trace amplitude for $\mathrm{PcP}$ and $\mathrm{PKiKP}$ and their ratio are shown in Fig. 13, for two azimuthal windows $310^{\circ}-330^{\circ}$ (western US) and $330^{\circ}-347^{\circ}$ (Texas). Note that the amplitudes of PcP for Texas are behaving PREM-like while PKiKP (black) jumps up starting near $30^{\circ}$. Thus, the ratio of PKiKP/PcP becomes over a factor of two larger as well. Also, note the large difference in PKiKP between these events beneath Nebraska. If these patterns are controlled by the ICB, we should see a better match by simply projecting these dots downward as in Fig. 14. The combined datasets display a strong edge roughly along the heavy dotted line separating the two colors where the amplitude of PKiKP jumps by a factor of two to three. More data are needed to develop a clearer image but such behavior can be explained by a Gaussian bump containing a transition from outer core to inner core, as shown in Fig. 11, at the edge of this boundary.

\section{Discussions}

The enigmatic nature of PKiKP observability is well known and confirmed in this study where its detection is low except for a few events. As in previous studies, we assume that both PcP and PKiKP have similar source strength, and the rapid variation (small scale) in each is caused by scattering (Tkalcic et al. 2009). Our numerical experiments suggest several approaches of data processing to help circumvent some of these problems and separate features caused by the upper mantle, CMB, and ICB.

First, we demonstrate that our earlier developed MPD analysis proves to be effective in processing $\mathrm{PcP}$ and PKiKP to obtain accurate and systematical measures of timing and amplitude. Whereas processing long-period waveform data displays a clear relationship between amplitude and complexity, Sun and Helmberger (2011), short-period data does not. This feature is confirmed by numerical experiments on random media. But given the rapid changes in upper-mantle scattering, it proved difficult to find systematic variation in differential PKiKP-PcP times as in the Dai et al. (2012) study. However, changes in $\mathrm{PcP}$ and PKiKP amplitude due to deep structure can be detected either by measuring the ratio of amplitudes from two neighboring events or by stacking. This approach works for numerical experiments with combinations of deterministic features at the core boundaries and random scatterers near the Earth's surface. The data shows rapid changes in PKiKP amplitude that can be explained by gradational bumps on the inner core with heights of several $\mathrm{kms}$ and lengths one degree horizontally, similar to that found along paths beneath the Banda Sea to Japan, Dai et al. (2012), and suggested by Cao et al. (2007). The latter paper addresses record sections of PKIKP and PKiKP, see Fig. 1, at distance of $135^{\circ}-140^{\circ}$ for earthquake doublets recorded by the Yellowknife Array. They suggest that a bump on the ICB must be involved, and that this bump changes its position with time, i.e., Song and Richards (1996), and causes the observed differences.

Previous study reveals that PKIKP and PKiKP bifurcation are distinctly different between the Western Hemisphere and the Eastern Hemisphere, although the boundaries are not well sampled, i.e., Niu and Wen (2001). Timing differences of up to $0.5 \mathrm{~s}$ with earlier bifurcations in the east and smaller PKIKP/PKiKP amplitudes are observed. While the average difference can be modeled well in 1D, Wen and Niu (2002), significant scatter (up to $50 \%)$ remains in any one record section. Note that the ray paths are essentially identical in the mantle, see Fig. 1, so that this type of variation must be caused in the deep earth. Here, we present synthetic predictions for a simple Gaussian-shaped bump centered near the PKiKP bounce point. Surprisingly, synthetics show that this feature, at distance 

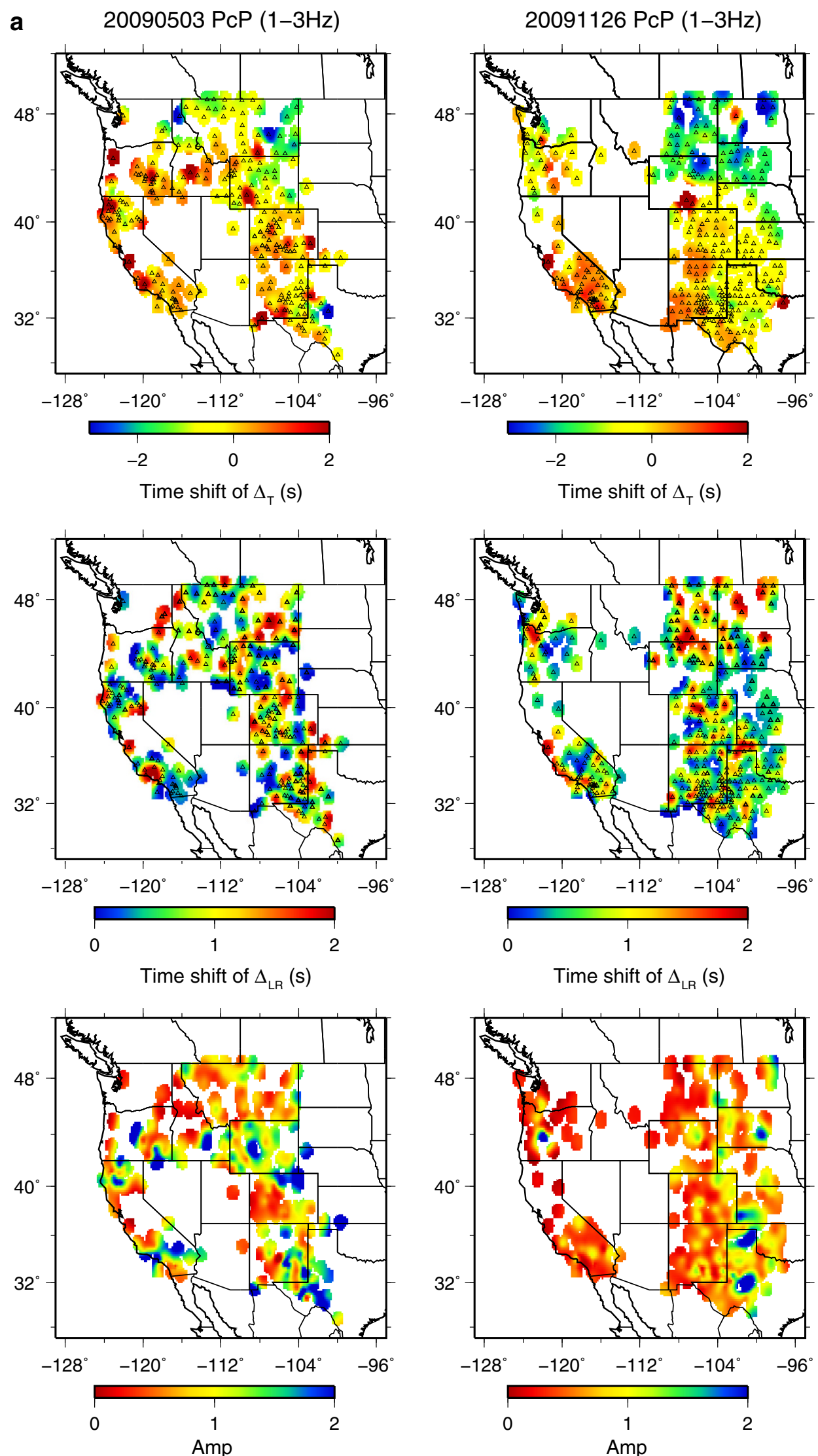

Fig. 16 MPD patterns of (A) PcP and (B) PKiKP phases for events 20090503 (A1) and 20091126 (A2), displaying considerable scatters 
b 2000503 PKiKP $(1-3 \mathrm{~Hz})$

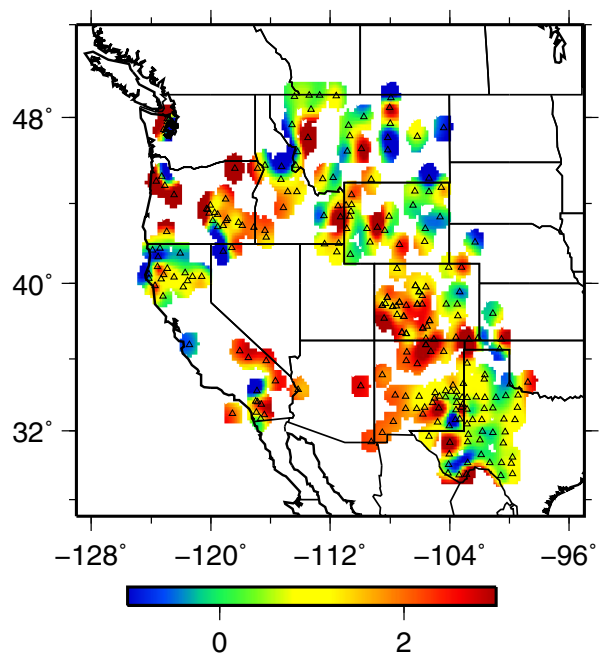

Time shift of $\Delta_{T}(\mathrm{~s})$
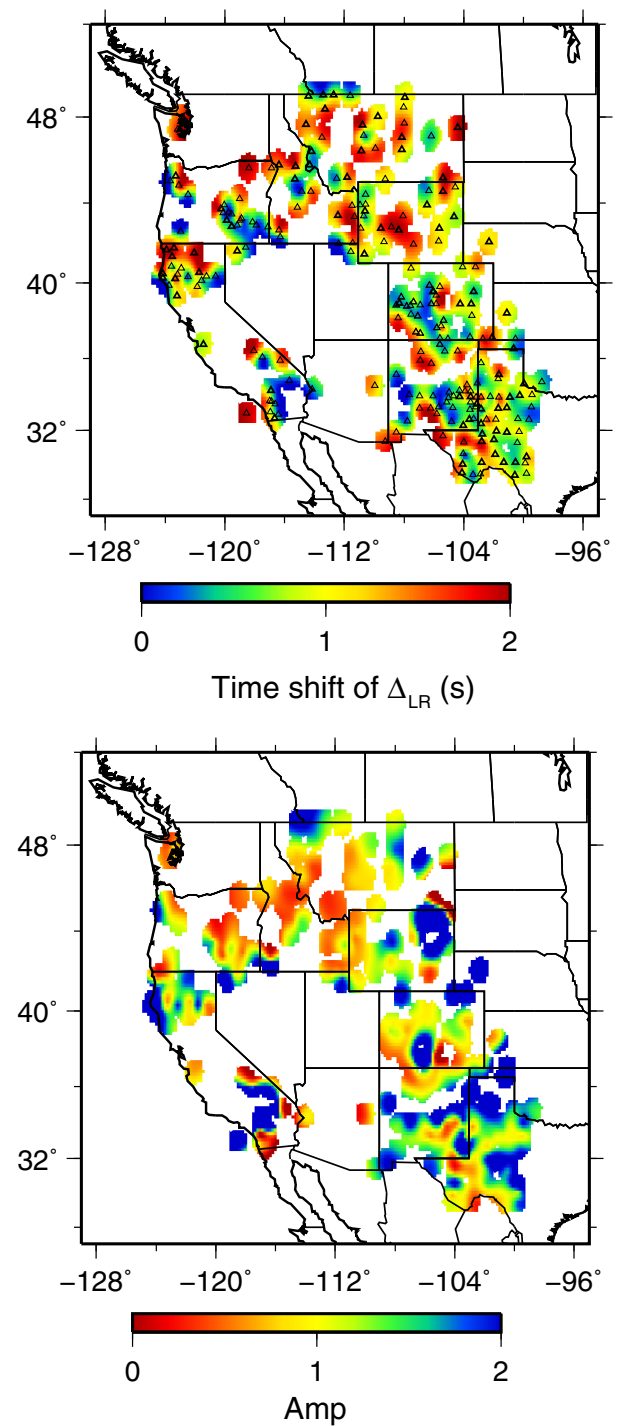

20091126 PKiKP (1-3Hz)

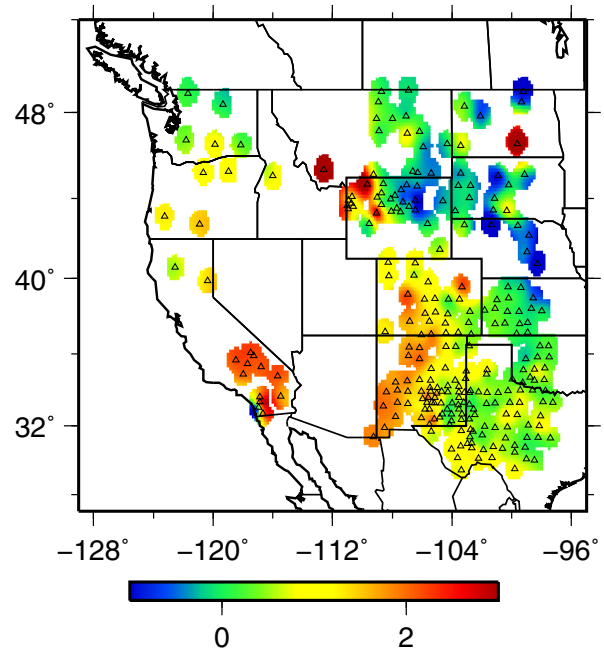

Time shift of $\Delta_{\mathrm{T}}(\mathrm{s})$
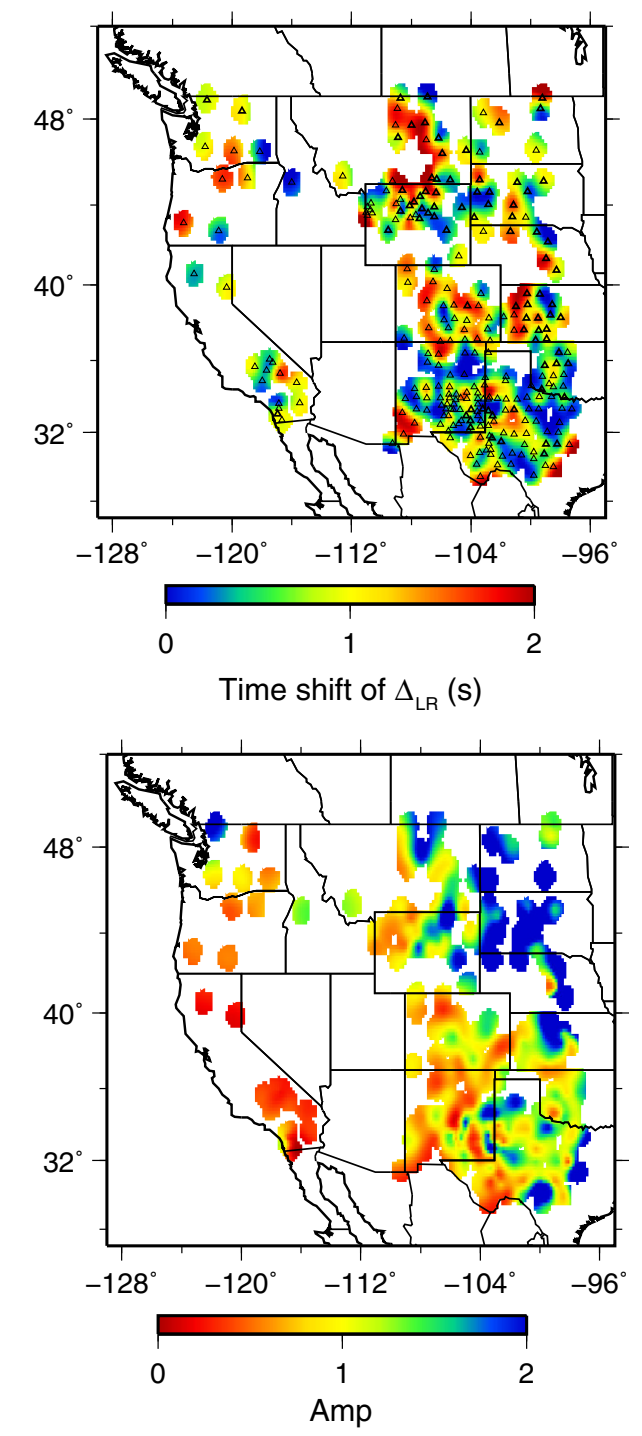

Fig. 16 continued 
around $130^{\circ}$, shortens the timing separation between PKIKP and PKiKP just by a few tenths of second and slightly changes the amplitude ratio, see Fig. 24. Comparing typical anomalies at the ICB and CMB in Fig. 15, we can see CMB proves more effective in producing distortions of the PKP bifurcation at the level commonly observed Wen and Niu (2002). In short, the role of CMB boundary complexity on PKiKP appears to be less important at small distances than that at large distance. Thus, to validate such fine-structure on the ICB, we need to sample both ICB and $\mathrm{CMB}$ with combinations of differential phases. This may be possible given the ever-expanded station coverage.

a
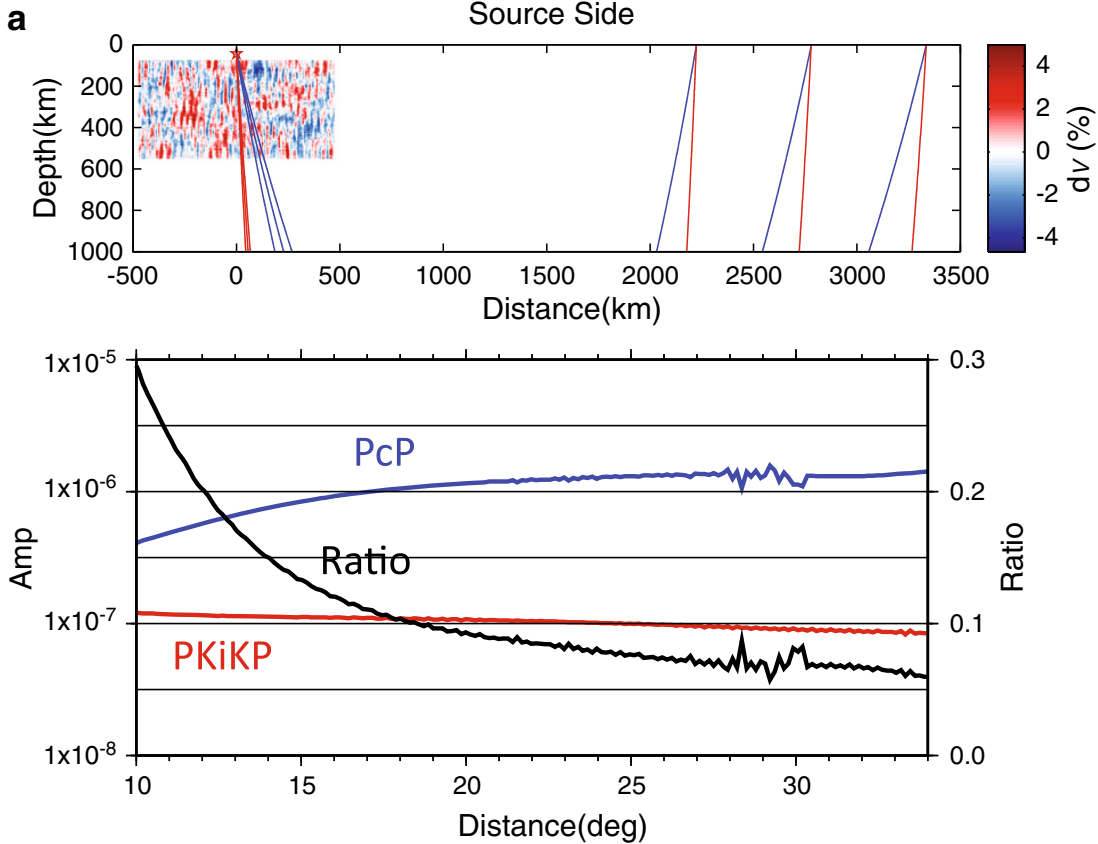

b
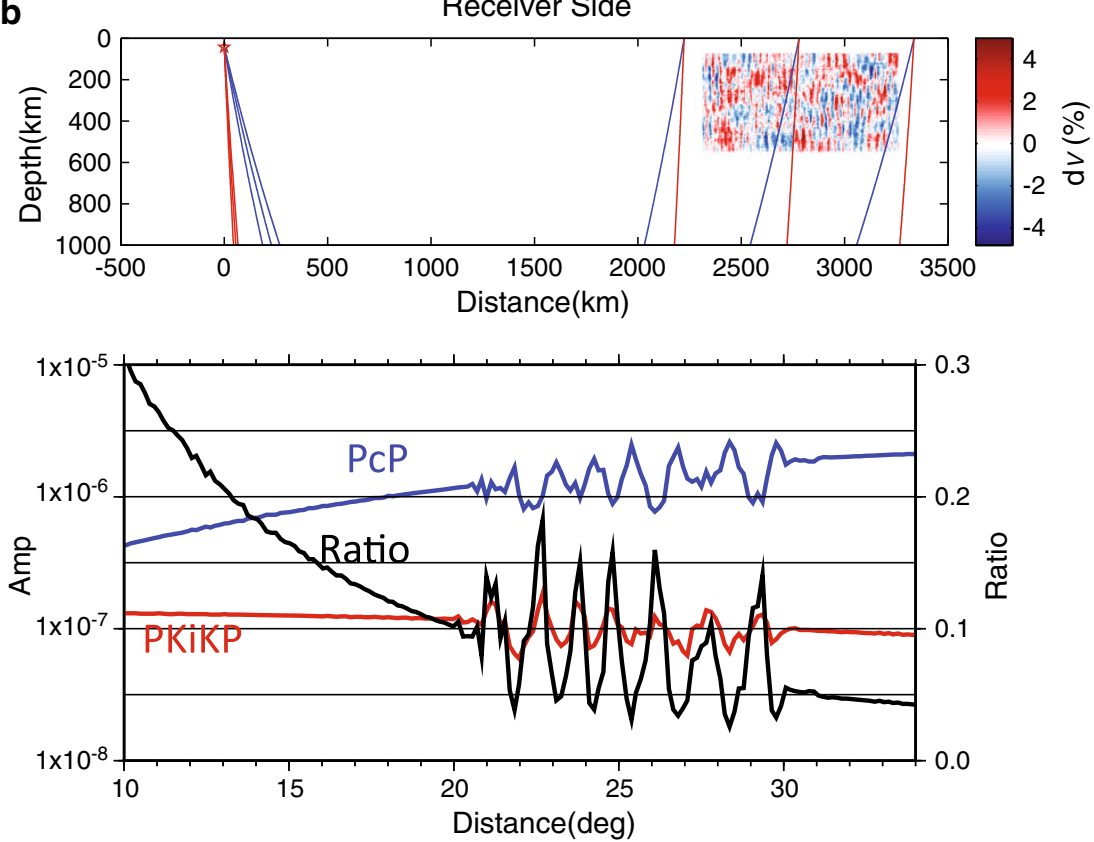

Fig. 17 Sensitivity testing where random scatter media are placed beneath the source side (a) and receiver side (b). The amplitude of PcP (red), $\mathrm{PKiKP}($ blue $)$ and their ratio (black) are also displayed. Note the scatters in the source side have small effects on the relative amplitude at the receivers, where the scatters at the receiver side have large effects 

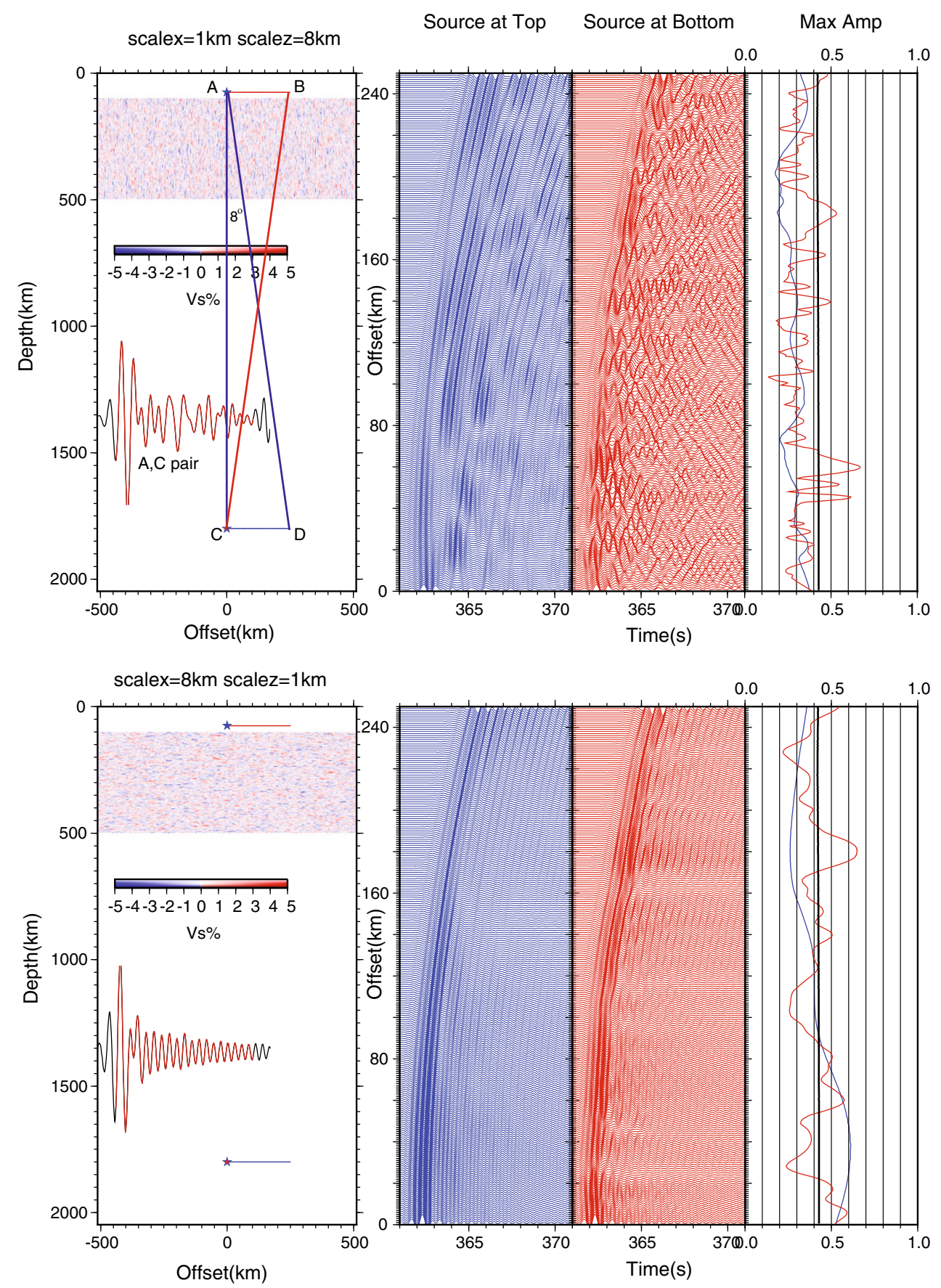

Fig. 18 For $\mathrm{SH}$ wave and point force source, we calculate synthetics for homogeneous model $\left(v_{\mathrm{S}} 4.8 \mathrm{~km} / \mathrm{s}\right)$ with top part having random variation. Two random media models with different scale length ratio are shown here. For each simulation, central frequency is $1.5 \mathrm{~Hz}$, grid space is $0.5 \mathrm{~km}$, which ensures 6 grid per wavelength. We put (1) Source at A, and receiver along CD line, (2) source at C, receiver along AB. The models are displayed on the left with synthetic sections and amplitudes on the right, color coded the same as source. The A,C pair seismogram satisfies reciprocity quite well, as shown in the inset. The record sections, AB, CD differs because of the different path in sampling the media. Variation in AB line is larger and more rapid than variation along CD line. Thus heterogeneity at source side has small effects on records, while heterogeneity at receiver side has large effects

\section{Conclusions}

In summary, we used USArray data to study complexity of the ICB interface. Following previous efforts, we used two neighboring events method and local stacking method to suppress scattering from shallow receiver structure. We find numerical simulation is especially useful to help us understand where in the earth the complexities are likely to be 


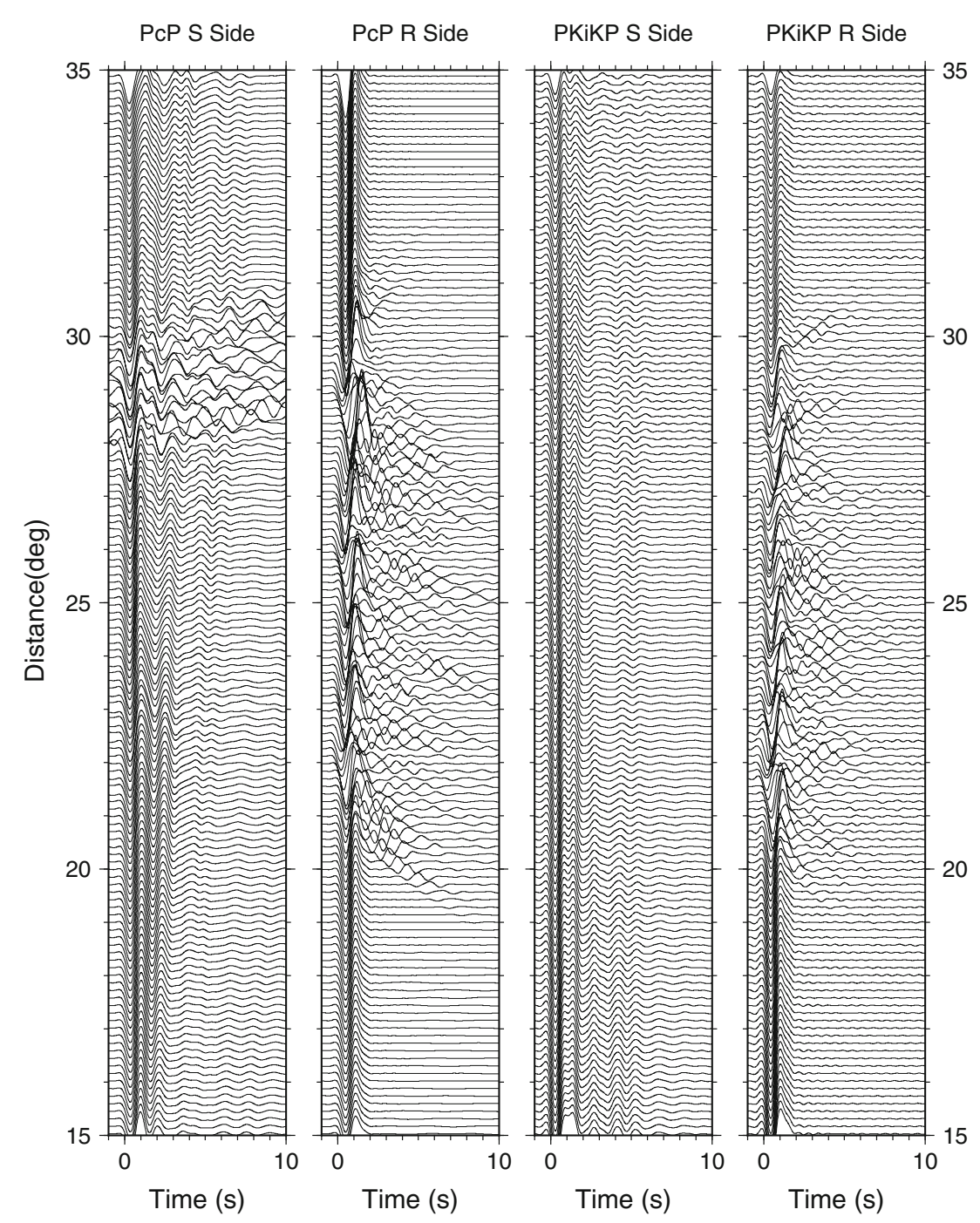

Fig. 19 Synthetic seismograms for core phases for heterogeneity at source side and receiver side. The interruption of PcP in the leftmost panel is due to surface wave. In general, note that heterogeneity at source side has small effects on records, while heterogeneity at receiver side has large effects

produced. We found that local stacking of synthetics $(>5$ stations with two degree) largely reduces receiver effects, even in case of very strong scattering, and then deep structure can be identified. The data was processed using this "rolling stacks" to generate a relative amplitude disk for each location and then migrated to the ICB. Such a map at ICB shows sharp edges where amplitudes change by a factor of 2-3. Simulations of 2D structures suggest changes in the ICB, either a sudden increase of transitional thickness (a few km high of patchy mushy layer) and/or a sharp horizontal change in ICB elevation, can produce such effects.

Acknowledgments We thank the Editor and two anonymous reviewers for their comments which greatly improved the manuscript. We also thank Brandon Schmandt for introducing us to these PKiKP data. Data were provided by the IRIS data center and Earthscope USArray. This work was supported by NSF EAR-1053064 and CSEDI EAR-1161046 at CalTech with partial support of D. Sun at USC under EAR-0809023.

\section{Appendix 1}

\section{Waveform Complexity Processing}

The travel times $\Delta_{\mathrm{T}}$ are the most stable showing the relatively slow Western United States (WUS). Sun and Helmberger (2011) show that at long-period, $\Delta_{\mathrm{LR}}$ maps generally correlates with the amplitude map, i.e., large $\Delta_{\mathrm{LR}}$ indicates strong multi-pathing and has low amplitude. Fig. 16 shows that some features following this pattern, but they do not match very well. It appears that the strong attenuation beneath WUS is an additional feature. These same complexities appear in the PKiKP maps but are not well correlated with what is observed in the PcP maps. Here, the overall amplitudes and $\Delta_{\mathrm{LR}}$ pattern agree better for PKiKP. Note that the amplitudes scatter about as much as in earlier $m_{\mathrm{b}}$ bias measures, Lay and Helmberger (1983a, b), used in studying attenuation. They 
found that while the western US has lower amplitude than eastern US with a bias $\delta m_{\mathrm{b}}$ of 0.26 (corresponding an average amplitude difference of 2), any one station sampled in either region can differ by this amount. Here is a much larger sample but generally in agreement. The average jump in S-wave travel times is about 6s, Grand and Helmberger (1984), Lay and Helmberger (1983a, b) and about half of this for P-waves. These features occur roughly along the Rocky Mountain Front. While PcP results displayed in Fig. 16 are in general agreement with these earlier studies, the PKiKP amplitude near the new Mexico-Texas boundary for event 20091126 are anomalously low. We think this is caused by the ICB as discussed later.

One of the remarkable fine-scale features of this mapping is the behavior of amplitudes between PcP and PKiKP along the northwestern coast, especially for event 20091126. Note the reversed strength between $\mathrm{PcP}$ and PKiKP, i.e., when PcP is weak, PKiKP is strong. We also found extreme differences in PKiKP amplitudes at neighboring stations, less than $50 \mathrm{~km}$ apart, where one is in the noise while the other is very strong, see Fig. 21. These observations appear to be related to the subduction zone as in the Tkalcic et al. (2010) study. However, most of stations in our datasets do not display such anti-correlations. The variation appears to be caused by upper mantle scattering as is known to some degree, i.e., Nielsen et al. (2003).

\section{Appendix 2}

\section{Waveform Simulation}

Significant progress in 3D modeling of global seismograms at the longer periods (17-100 s) becomes possible with the development of advanced computing systems, Komatitsch and Tromp (2002). Extending this to shorter periods is challenging because of computing demands. So hybrid techniques prove useful. Several axisymmetric methods can reach shorter periods such as Nissen-Meyer et al. (2007). The 2D pseudo-spectral approach as described in Cormier (2000) is particularly good at treating random media and used extensively in the recent study by Tkalcic et al. (2009). Some hybrid methods use a combination of analytic and finitedifference (FD) methods which are interfaced with the Kirchhoff integral. Essentially, one propagates a signal through simple regions using generalized rays and only uses numerical methods in the heterogeneous regions, Wen and Helmberger (1998). This method was used by Dai et al. (2012) in inserting a box-like structure at the ICB. We have reproduced their results using our new 2D FD code, Li et al. (2014), as discussed in the main text.

To obtain seismograms from a point source in $2 \mathrm{D}$ media, we need to consider in-plane propagation and out-of-plane spreading. Three difficulties needs to be addressed in implementing this procedure: (1) the mapping procedure involved in 3D source excitation for earthquakes; (2) 3D spreading corrections; and (3) reducing the spherical earth to a flattened model. We solved the first issue by using a modern moment tensor excitation approach. The out-of-plane geometric spreading is accounted for by applying a post-simulation filter. In addition, an earth-flattening transformation is used to obtain simulations in a spherical geometry using calculations based on Cartesian coordinates. Simulations are generated using the 2D staggered grid finite difference method on graphics processing units (GPUs), which proves to be highly efficient and flexible for modeling global seismograms, including core phases. The effectiveness of this method is demonstrated by comparing our synthetics with the frequency-wavenumber normal-mode and SEM synthetics, Li et al. (2014).

The 2D pseudo-spectral method has been used extensively for studying scattering effects near the CMB, i.e., Cormier (2000). Recently it was used to investigate receiver effects for plane-wave incidence of $\mathrm{PcP}$ and PKiKP, Tkalcic et al. (2010). We repeated this experiment but assumed a point source for the PREM model, Fig. 17. We place the "scattering boxes" either beneath the source or the receivers. We assume an explosion source and Gaussian random media. To test our code, we conducted a detailed reciprocity numerical experiment given in Fig. 18. In Fig. 17, we can see ray paths for PKiKP leaving the source region are nearly the same for receivers from $20^{\circ}$ to $30^{\circ}$ degree. The PcP rays are also compact but have an offset relative to PKiKP. In contrast, the ray paths are well separated at the receiver side with each path encountering a distinct structure. The synthetics for these two situations are given in Fig. 19 and the amplitudes variation is included in Fig. 17. Note the rapid amplitude decay of PKiKP and the gentle increase in PcP controlled by the PREM model which is similar to the AK135 results as shown in Tkalcic et al. (2009). The PKiKP/PcP ratio is also displayed where the effect from source side scattering (Fig. 17) is smaller than that from receiver side scattering.

\section{Appendix 3}

See Figs. 20, 21, 22, 23, 24. 


\section{Velocity BHZ}

Time(s)

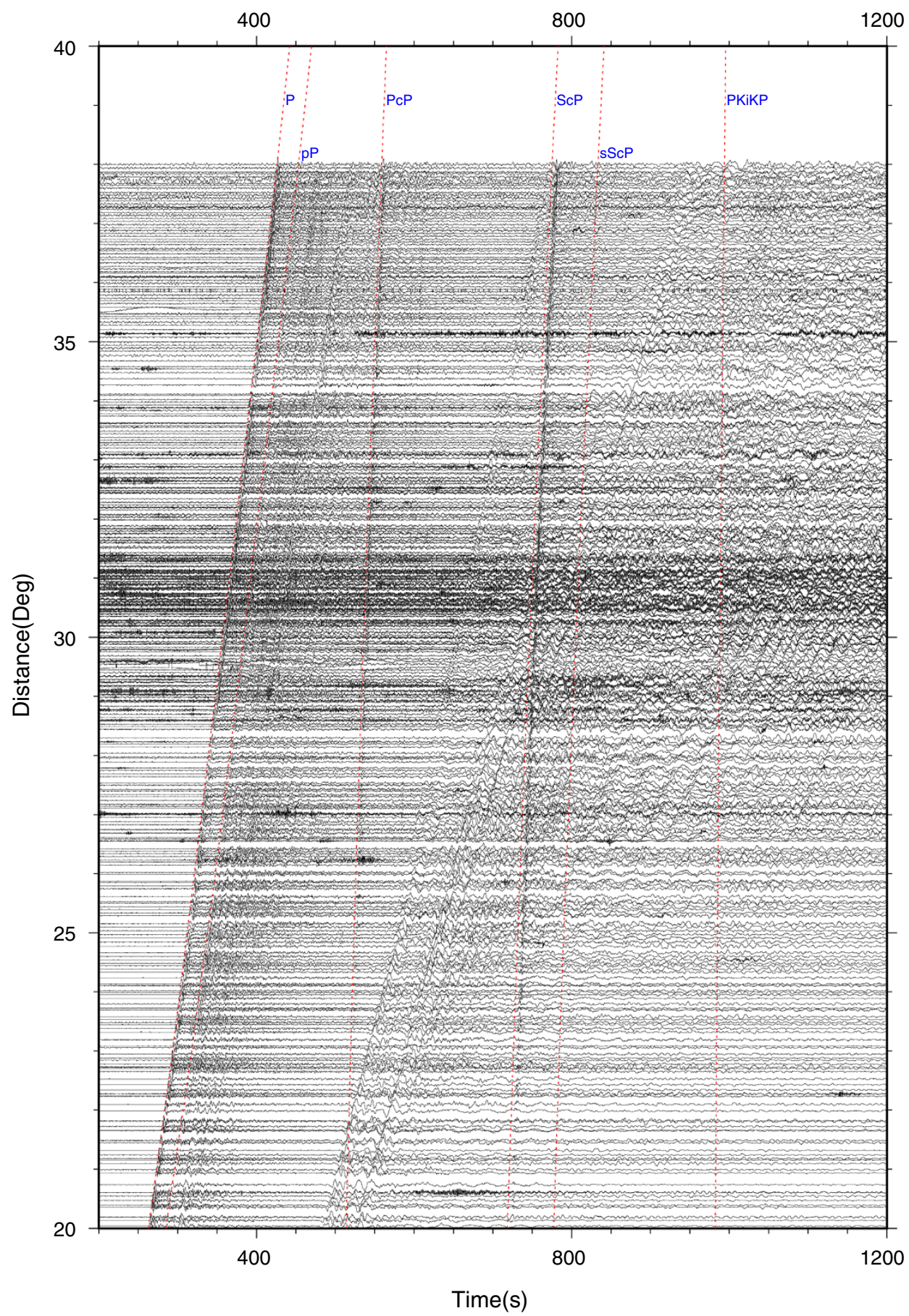

Fig. 20 (a) Broadband velocity seismogram record section for the 20090503 event. (b) Applying bandpass filter from 1 to $3 \mathrm{~Hz}$ enhances the PKiKP phase as in Fig. 2 


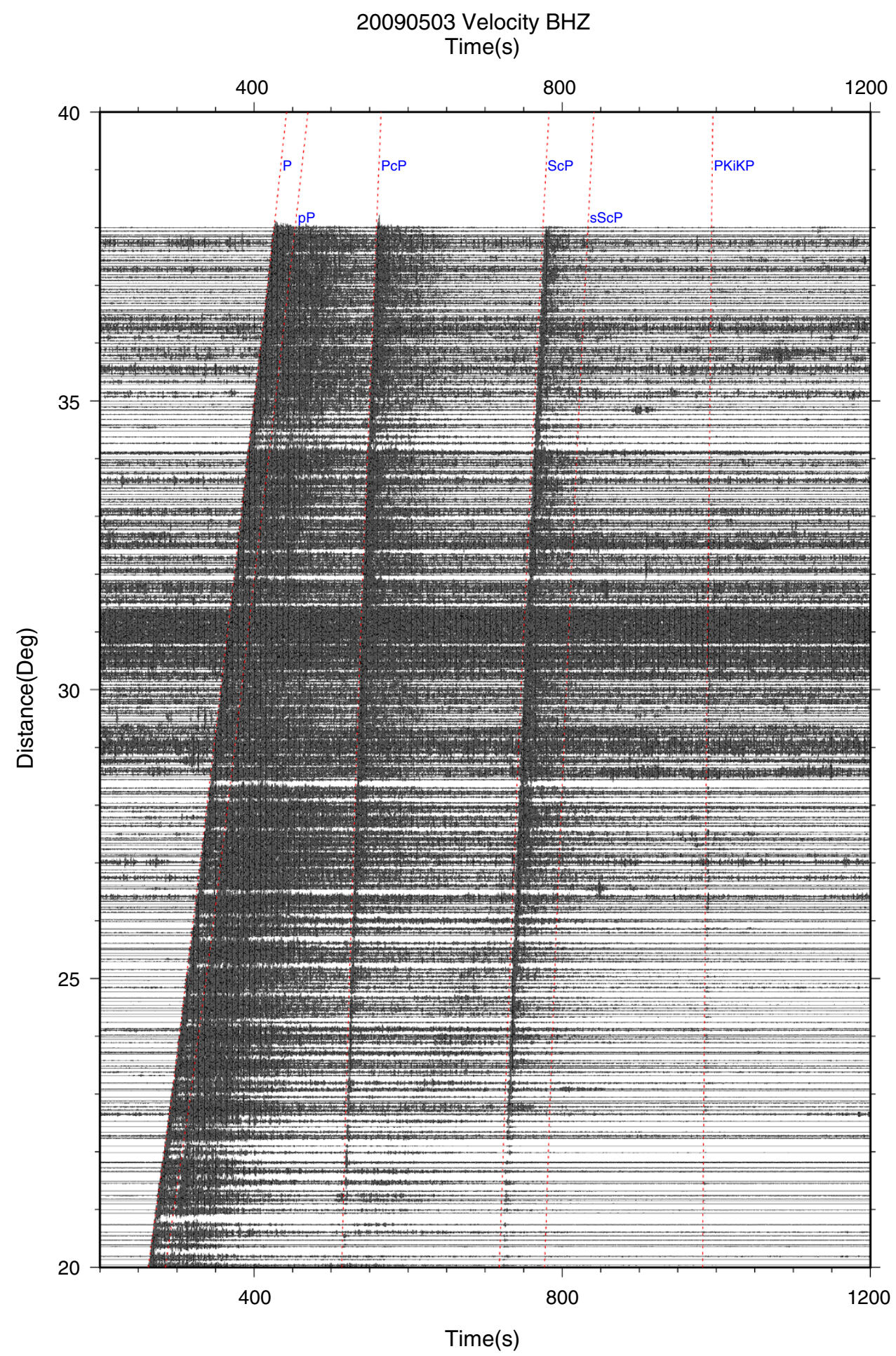

Fig. 20 continued 


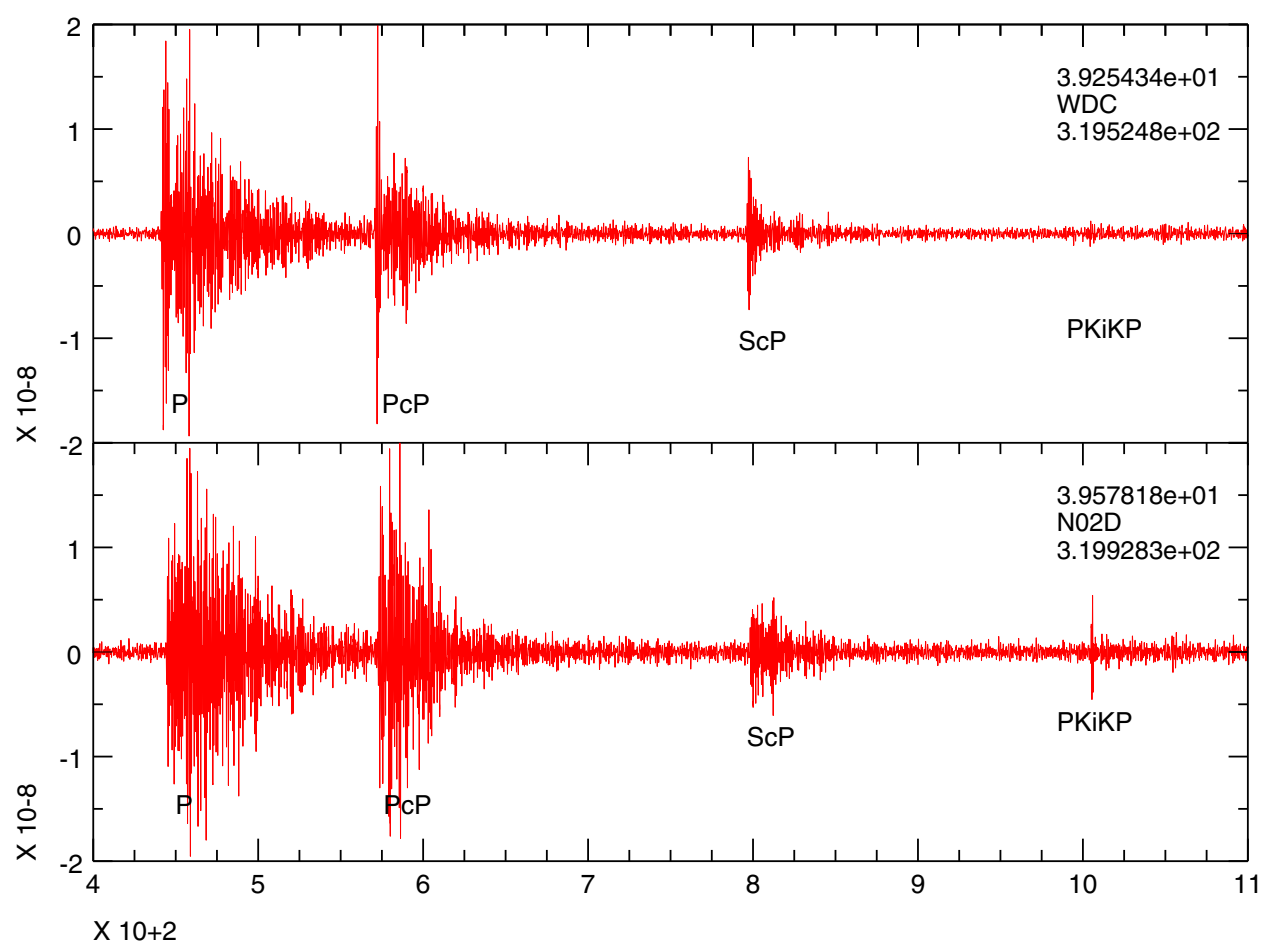

Fig. 21 Seismograms (1 to $3 \mathrm{~Hz}$ ) of 20091126 event for two northern California stations $45 \mathrm{~km}$ apart, where the core phases ray may sample the subducted slab. Note that $\mathrm{P}, \mathrm{PcP}$ and $\mathrm{ScP}$ phases are about the same amplitude, while PKiKP amplitudes are quite different 


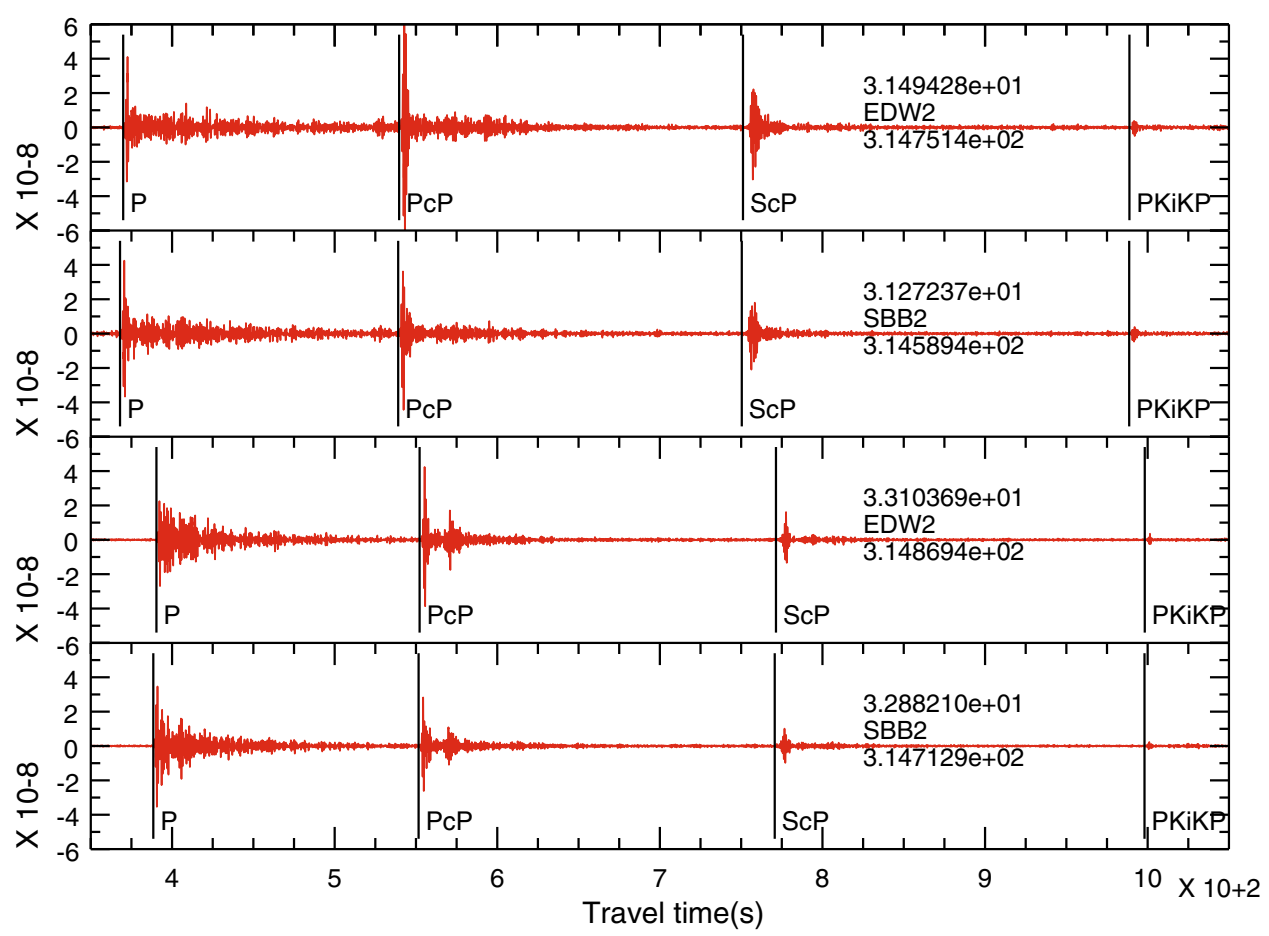

$$
0503 \text { EDW2 }
$$

Fig. 22 A case where PKiKP/PcP ratio of ratio has a factor of 2 difference between nearby stations, see Fig. 8 . Seismograms (1 to $3 \mathrm{~Hz}$ ) of each phase are displayed in the lower panel. Black number indicates maximum absolute amplitude for each phase window 
wig2lambda050

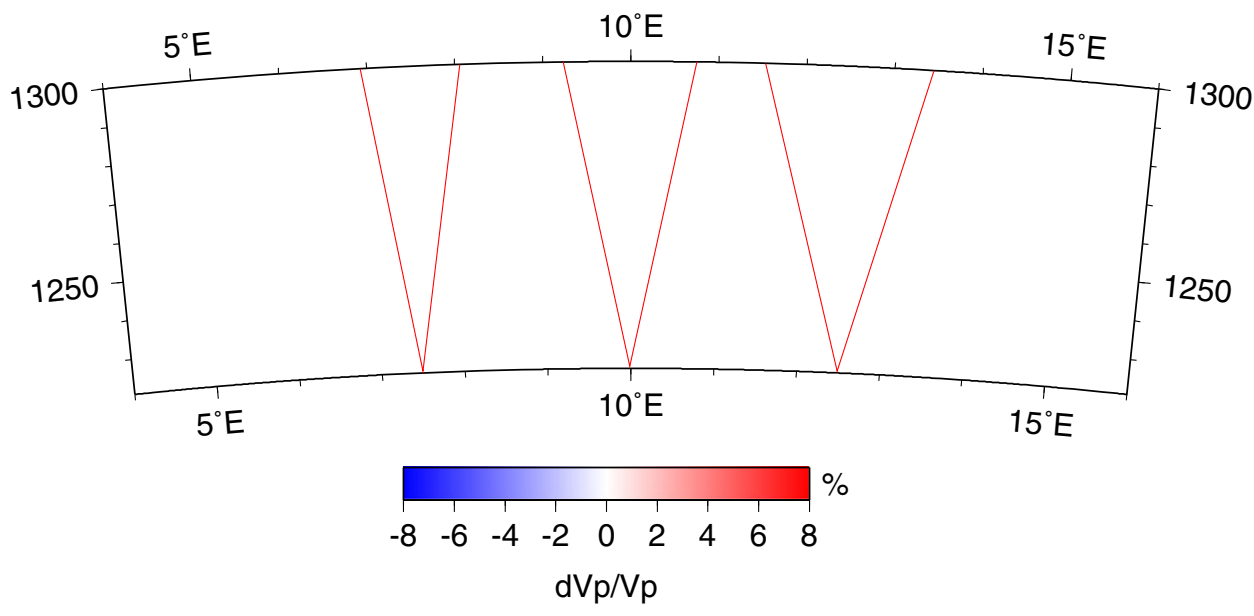

wig2lambda050.ps pkikp(blue) and precursor(red), prem(black)

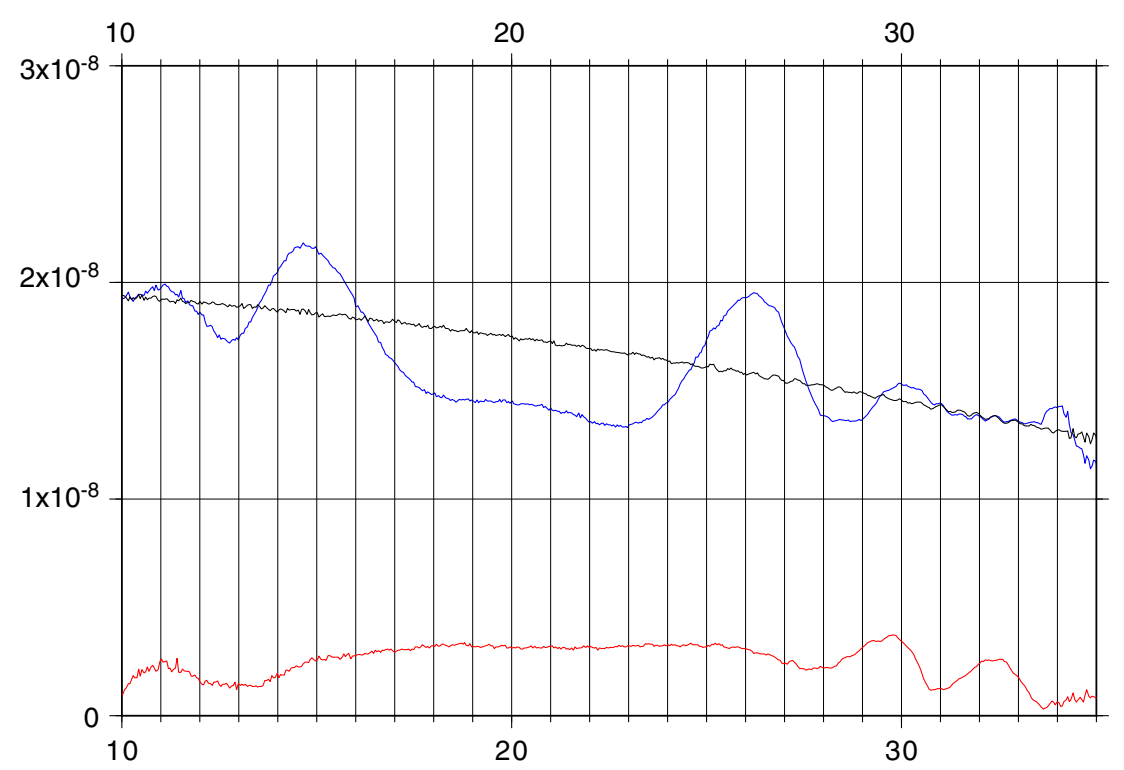

Fig. 23 A model similar to the first model in Fig. 12, but with heterogeneity at ICB of $14 \mathrm{~km}$ and about $0.5^{\circ}$ width 


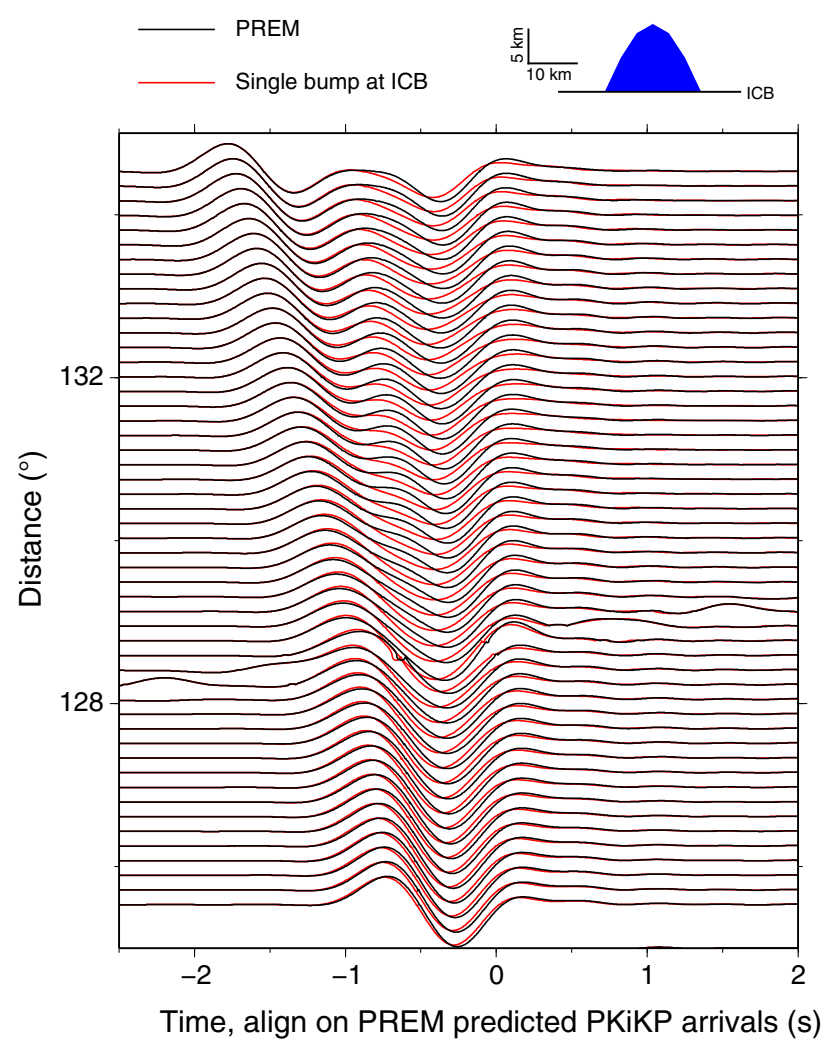

Fig. 24 PKiKP and PKIKP synthetics for the PREM model (black) and the model with a single bump at the ICB (red). The bump has the width of $20 \mathrm{~km}\left(1^{\circ}\right)$ and the height of $10 \mathrm{~km}$ with the inner core properties. The disruption near $128^{\circ}$ is another secondary phase interfering with PKP system

\section{References}

Adushkin VV, Ovchinnikov VM (2004) The mosaic in reflections from the Earth's solid core boundary. Dokl Earth Sci 397(6):883-885

Cao AM, Masson Y, Romanowicz B (2007) Short wavelength topography on the inner-core boundary. Proc Natl Acad Sci USA 104:31-35

Cormier VF (2000) D as a transition in the heterogeneity spectrum of the lowermost mantle. J Geophys Res 105:16193-16205

Cormier VF, Choy GL (1986) A search for lateral heterogeneity in the inner core from differential travel times near PKP-D and PKP-C. Geophys Res Lett 13:1553-1556

Cormier VF, Richards PG (1977) Full wave theory applied to a discontinuous velocity increase: the inner core boundary. J Geophys 43:3-31

Cummins P, Johnson LR (1988) Short-period body wave constraints on properties of the Earth's inner core boundary. J Geophys Res 93:9058

Dai ZY, Wang W, Wen LX (2012) Irregular topography at the Earth's inner core boundary. Proc Natl Acad Sci USA 109:7654-7658

Engdahl ER, Flinn EA, Romney CF (1970) Seismic waves reflected from the Earth's inner core. Nature 228:852-853

Grand SP, Helmberger DV (1984) Upper mantle shear structure of North America. Geophys J Roy Astron Soc 76:399-438
Helmberger DV, Ni S (2005) Approximate 3D body-wave synthetics for tomographic models. Bull Seismol Soc Am 95:212-224

Kawakatsu H (2006) Sharp and seismically transparent inner core boundary region revealed by an entire network observation of near-vertical PKiKP. Earth Planets Space 58:855-863

Komatitsch D, Tromp J (2002) Spectral-element simulations of global seismic wave propagation-II. Three-dimensional models, oceans, rotation and self-gravitation. Geophys J Int 150:303-318

Koper KD (2004) Observations of PKiKP/PcP amplitude ratios and implications for Earth structure at the boundaries of the liquid core. J Geophys Res 109:B03301

Koper KD, Dombrovskaya M (2005) Seismic properties of the inner core boundary from PKiKP/P amplitude ratios. Earth Planet Sci Lett 237:680-694

Lay T, Helmberger DV (1983a) Body-wave amplitude and traveltime correlations across North America. Bull Seismol Soc Am 73:1063-1076

Lay T, Helmberger DV (1983b) A shear velocity discontinuity in the lower mantle. Geophys Res Lett 10:63-66

Li DZ, Helmberger DV, Clayton RW, Sun DY (2014) Global synthetic seismograms using a 2D finite difference method. Geophys J Int 197:1166-1183

Nielsen L, Thybo H, Levander A, Solodilov LN (2003) Origin of upper-mantle seismic scattering - evidence from Russian peaceful nuclear explosion data. Geophys J Int 154:196-204

Nissen-Meyer T, Fournier A, Dahlen FA (2007) A two-dimensional spectral-element method for computing spherical-earth seismograms-I. Moment-tensor source. Geophys J Int 168:1067-1092

Niu F, Wen L (2001) Hemispherical variations in seismic velocity at the top of the Earth's inner core. Nature 410:1081-1084

Poupinet G, Pillet R, Souriau A (1983) Possible heterogeneity of the Earth's core deduced from PKIKP travel times. Nature 305:204-206

Shearer P, Masters G (1990) The density and shear velocity contrast at the inner core boundary. Geophys J Int 102:491-498

Shimizu H, Poirier JP, Le Mouël JL (2005) On crystallization at the inner core boundary. Phys Earth Planet Inter 151:37-51

Song X, Helmberger DV (1992) Velocity structure near the inner core boundary from waveform modeling. J Geophys Res 97:6573-6586

Song X, Richards PG (1996) Seismological evidence for differential rotation of the Earth's inner core. Nature 382:221-224

Sun D, Helmberger D (2011) Upper-mantle structures beneath USArray derived from waveform complexity. Geophys J Int 184:416-438

Sun DY, Helmberger DV, Jackson JM, Clayton RW, Bower DJ (2013) Rolling hills on the core-mantle boundary. Earth Planet Sci Lett 361:333-342

Tkalčić H, Kennett BLN, Cormier VF (2009) On the inner-outer core density contrast from PKiKP/PcP amplitude ratios and uncertainties caused by seismic noise. Geophys J Int 179:425-443

Tkalčić H, Cormier VF, Kennett BLN, He K (2010) Steep reflections from the earth's core reveal small-scale heterogeneity in the upper mantle. Phys Earth Planet Inter 178:80-91

Waszek L, Irving J, Deuss A (2011) Reconciling the hemispherical structure of Earth's inner core with its super-rotation. Nat Geosci 4:264-267

Wen L (2006) Localized temporal change of the Earth's inner core boundary. Science 314:967-970

Wen L, Helmberger DV (1998) A two-dimensional P-SV hybrid method and its application to modeling localized structures near the core-mantle boundary. J Geophys Res 103:17901-17918

Wen L, Niu F (2002) Seismic velocity and attenuation structures in the top of the Earth's inner core. J Geophys Res 107:2273 